ARTICLE

\title{
A resilient formin-derived cortical actin meshwork in the rear drives actomyosin-based motility in 2D confinement
}

\author{
Nagendran Ramalingam ${ }^{1, \star, \dagger}$, Christof Franke ${ }^{2, \star}$, Evelin Jaschinski ${ }^{3}$, Moritz Winterhoff ${ }^{2}$, Yao Lu $^{4}$, \\ Stefan Brühmann², Alexander Junemann², Helena Meier ${ }^{2}$, Angelika A. Noegel ${ }^{5}$, Igor Weber ${ }^{6}$, \\ Hongxia Zhao ${ }^{4}$, Rudolf Merkel $^{3}$, Michael Schleicher ${ }^{1} \&$ Jan Faix ${ }^{2}$
}

Cell migration is driven by the establishment of disparity between the cortical properties of the softer front and the more rigid rear allowing front extension and actomyosin-based rear contraction. However, how the cortical actin meshwork in the rear is generated remains elusive. Here we identify the mDia1-like formin A (ForA) from Dictyostelium discoideum that generates a subset of filaments as the basis of a resilient cortical actin sheath in the rear. Mechanical resistance of this actin compartment is accomplished by actin crosslinkers and IQGAP-related proteins, and is mandatory to withstand the increased contractile forces in response to mechanical stress by impeding unproductive blebbing in the rear, allowing efficient cell migration in two-dimensional-confined environments. Consistently, ForA supresses the formation of lateral protrusions, rapidly relocalizes to new prospective ends in repolarizing cells and is required for cortical integrity. Finally, we show that ForA utilizes the phosphoinositide gradients in polarized cells for subcellular targeting.

\footnotetext{
${ }^{1}$ Anatomy III/Cell Biology, BioMedCenter, Ludwig-Maximilians-University, Grosshaderner Str. 9, Planegg-Martinsried, Germany. ${ }^{2}$ Institute for Biophysical Chemistry, Hannover Medical School, Carl-Neuberg-Strasse 1, Hannover 30625, Germany. ${ }^{3}$ Institute of Complex Systems, ICS-7: Biomechanics, Forschungszentrum Jülich GmbH, Jülich 52425 Germany. ${ }^{4}$ Institute of Biotechnology, University of Helsinki, PO Box 56, Helsinki 00014, Finland. ${ }^{5}$ Center for Biochemistry, Medical Faculty, University of Cologne, Köln 50931, Germany. ${ }^{6}$ Division of Molecular Biology, Ruder Bošković Institute, Bijenička 54, Zagreb 10000, Croatia. * These authors contributed equally to this work. † Present address: Department of Neurology, Columbia University, 650 West 168 th Street, New York 10032, New York, USA. Correspondence and requests for materials should be addressed to J.F. (email: faix.jan@mh-hannover.de).
} 
C ell migration is implicated in numerous processes in normal physiology and disease ${ }^{1,2}$. On the basis of their properties, eukaryotic cells can move by distinct modes of action. To delineate this heterogeneity, the behaviours of single cells have been subdivided into two main categories of mesenchymal or amoeboid migration modes depending on cell morphology, mechanisms of force generation, cytoskeleton organization and cell-substrate adhesion ${ }^{1,3}$. The rather slow mesenchymal mode of cell migration, as exemplified by fibroblasts, is characterized by strong cell-substrate adhesion, prominent stress fibres and extended formation of protruding lamellipodia or ruffles driven by Arp $2 / 3$ complex-mediated actin polymerization at the leading edge ${ }^{4}$. Fast amoeboid cell migration, as utilized by immune cells or Dictyostelium amoebae, is instead characterized by rounder shape, weaker adhesion, absence of stress fibres and formation of actin-rich pseudopods or hydrostatic pressure-driven blebs in their fronts and myosin-II-driven contractility in the rears ${ }^{5,6}$. Notably, these distinct motility modes are extremes of a broad spectrum characterized by smooth transitions. In addition, some cells, in particular cancer cells, exhibit plasticity and can switch from the mesenchymal to the amoeboid motility mode to drive invasion ${ }^{1}$.

The movement of cells is the final readout of multiple processes including actin assembly, adhesion and contractility, and entails the breaking of symmetry to generate a cell front and a cell rear along the axis in the direction of movement ${ }^{7}$. There is strong evidence that global actin-myosin network reorganization and non-muscle myosin-II-driven contraction initiate symmetry breaking by forming the imminent rear of the cell ${ }^{8}$, which restricts protrusions to the cell front. The transition from a semistable unpolarized state to a polarized migratory state can occur randomly, but can also be induced by mechanical stimulation, leading to an anisotropic distribution of the actomyosin system, which is subsequently sustained by positivefeedback loops ${ }^{9}$. Polarity can be additionally stabilized for instance in chemotactically migrating cells by extracellular cues ${ }^{10}$.

Cell membrane deformation is coupled to cortical tension and stiffness, membrane-cortex adhesion and hydrostatic pressure ${ }^{3,11}$. Micropipette aspiration (MPA) assays with polarized migrating Dictyostelium amoebae revealed easier deformability at the cell front as compared with the trailing edge, suggesting either weaker anchoring of the membrane to the underlying actin cytoskeleton or a less stiffer cortex in the leading edge ${ }^{12}$. Similar differences in the cortical properties have also been demonstrated for higher eukaryotes strongly suggesting that this disparity is a general requirement of actomyosin-driven or actomyosin-assisted cell migration ${ }^{3,13}$. The contractile actin cortex is a thin layer of bundled or crosslinked actin filaments, non-muscle myosin II and associated proteins beneath the plasma membrane of eukaryotic cells $^{11,14}$. Assembly and contraction of this layer generates cortical tension and plays a central role in migration ${ }^{7}$, cell division ${ }^{15}$ and tissue morphogenesis ${ }^{16}$. Despite its significance, the assembly, structural organization, membrane attachment and mechanics of the actin-rich cortex are still not well understood.

Even though numerous proteins can promote actin assembly, Arp $2 / 3$ complex and formins are the major actin nucleators in cells ${ }^{17}$. Active Arp $2 / 3$ complex creates branches on the sides of existing mother filaments to generate a dense actin meshwork as exemplified by the actin architecture of the leading edge ${ }^{18}$. Formins instead nucleate and elongate unbranched actin filaments to form the cytokinetic ring, yeast cables or filopodial bundles ${ }^{17}$. A subgroup referred as diaphanous-related formins (DRFs) is tightly regulated. In their autoinhibited form, these proteins fold on themselves and are inactive. Binding of Rhofamily GTPases to the N-terminal GTPase-binding domain
(GBD) releases this autoinhibition and renders the proteins active. Both, Arp2/3 complex and different DRFs have been implicated in the formation of actin cortex in various cell types, although most of the obtained evidence was rather indirect ${ }^{19-22}$. More recent work convincingly demonstrated a major contribution of Arp2/3 complex and the DRF mDial in generation of the cortical actin cytoskeleton in M2 and HeLa cells ${ }^{23}$. As revealed by this study, Arp $2 / 3$ complex and mDial contributed equally to F-actin formation in the cortex but had different effects on cortical integrity, blebbing and cell division. While for instance mDial depletion arrested cell division, depletion or perturbation of Arp $2 / 3$ complex by itself did not. However, Arp2/3 complex perturbation potentiated the effect of mDial depletion, suggesting that mDial and Arp2/3 play different roles in cortex function.

In this study, we aimed to identify actin assembly factors that contribute to the formation and function of the rear cortex of polarized, migrating Dictyostelium cells. Our results show that ForA-generated actin filaments provide the basis of a resilient cortical shield that is strengthened by actin crosslinkers and IQ-motif-containing GTPase-activating protein (IQGAP)-related proteins. This actin compartment is recruited to the rear by phosphoinositide gradients and allows withstanding high actomyosin-based contractility and guides hydrostatic pressure to the front facilitating cell migration in a two-dimensional (2D)confined environment. In addition, we reveal that this cortical network also suppresses the formation of unproductive lateral protrusions. Finally, our findings strongly suggest that ForA represents the functional homologue of $\mathrm{mDia}$ from higher eukaryotes.

\section{Results}

ForA localizes to the rear actin cortex of migrating cells. Since the hydrostatic pressure-driven blebs are a hallmark of migrating Dictyostelium cells in confined environments ${ }^{6}$, and the loss of myosin II is detrimental for cell migration ${ }^{24}$, we hypothesized specific actin assembly factors to exist in the rear that contribute to actomyosin-driven contractility. As the Arp $2 / 3$ complex is predominantly localized in the cell front, we systematically screened for the localization of as yet uncharacterized Dictyostelium formins by expression of green fluorescent protein (GFP) fusion proteins. We identified active ForA, lacking its C-terminal diaphanous-autoinhibitory domain (ForA $\triangle \mathrm{DAD}$ ), but not the autoinhibited full-length (FL) protein, to be strongly enriched in the rear cortex of migrating cells and the cleavage furrow of dividing cells, while it was evenly distributed in the cell cortex of unpolarized resting cells (Fig. 1a; Supplementary Fig. 1; Supplementary Movies 1 and 2). During random cell migration, vegetative Dictyostelium cells frequently generate multiple transient pseudopods along their periphery as opposed to developed chemotactically migrating cells that maintain a stable single front ${ }^{25}$. Interestingly, confocal timelapse imaging of randomly migrating ForA $\triangle \mathrm{DAD}$ cells revealed that birth of a new extension in the rear was faithfully associated with concomitant disappearance of the formin (Fig. 1b; Supplementary Movie 3), demonstrating that migratory cell protrusions are exclusively formed from cortical regions devoid of ForA. For $\mathrm{A} \triangle \mathrm{DAD}$ also always dynamically altered its position in spontaneous front-to-tail transitions of sharply turning cells (exemplarily shown in Fig. 1c; Supplementary Movie 4). Global measurement of cortical ForA fluorescence intensity around the entire cell periphery in these cells before, during and after repolarization revealed ForA accumulation in retracting zones before and after changing the direction of movement, while ForA intensity was evenly distributed in repolarizing cells (Fig. 1d; Supplementary Fig. 2). 
a

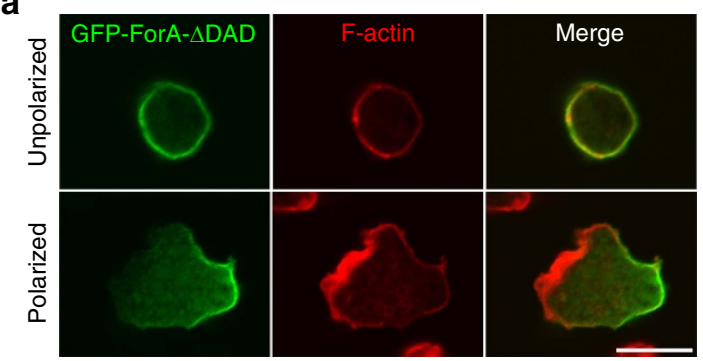

。

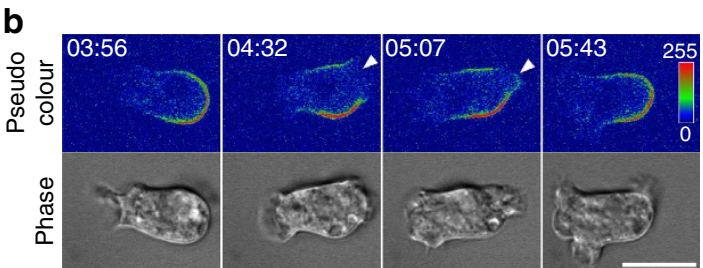

c

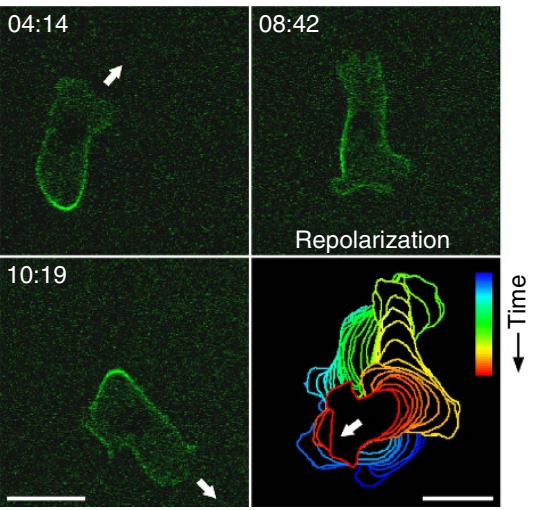

d

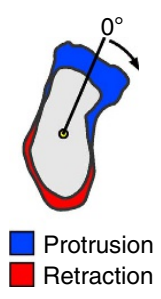

Before turning

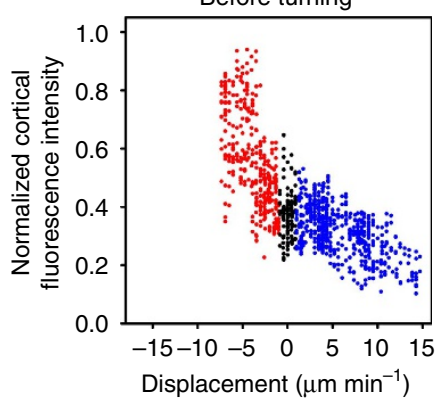

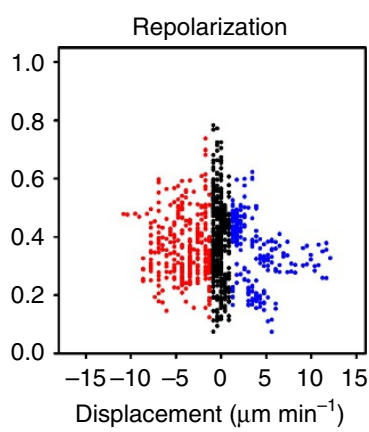

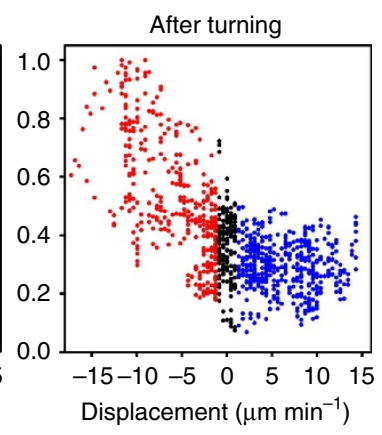

Figure 1 | Cortical localization of ForA. (a) GFP-tagged ForA $\triangle D A D$ expressed in for - $^{-}$cells localizes uniformly at the cortex of unpolarized cells but accumulates prominently at the trailing edge of polarized cells. Scale bar, $10 \mu \mathrm{m}$. (b) GFP-tagged ForA $\triangle \mathrm{DAD}$, depicted a by pseudo-coloured heatmap, instantly withdraws from the cortex before formation of lateral protrusions (arrowheads). Time corresponds to min:s and refers to Supplementary Movie 3 . Scale bar, $10 \mu \mathrm{m}$. (c) Relocation of active ForA in turning cells. The white arrow marks the direction of cell movement before and after repolarization and refers to Supplementary Movie 4. Colour-coded, stacked outlines of this cell during the turning event are shown in the low right corner. Scale bars, $10 \mu \mathrm{m}$. (d) ForA dynamically relocates to new prospective ends in repolarizing cells. Left: principle of radial displacement determination (for details see Methods). Plots show correlation between cortical ForA intensity and radial boundary displacement in retracting (red), non-dynamic (black) and protruding (blue) areas of three analysed cells before, during and after repolarization. In polarized, migrating cells ForA intensity was markedly increased in zones of high retraction at the rear and lower in protruding areas at the front. During repolarization, the edge displacements were smaller and unevenly distributed around the cell perimeter (see Supplementary Fig. 2c). Consistently, cortical ForA intensity was lower and homogenous at this stage.

ForA is regulated and promotes actin assembly. Canonical formins nucleate and elongate actin filaments, although their specific activities can be highly variable ${ }^{26}$. Thus, we analysed the biochemical properties of ForA in vitro after purification of a recombinant glutathione $S$-transferase (GST)-tagged C-terminal ForA fragment (ForA-C) encompassing five of the eight polyproline stretches from its $\mathrm{FH} 1$ domain followed by all of its C-terminal residues (aa 635-1,218, Fig. 2a). After removal of the GST-tag, ForA-C was first analysed in pyrene actin assays in which it stimulated actin polymerization in a concentrationdependent manner (Fig. 2b). Addition of the N-terminal regulatory region (ForA-FH3; Fig. 2a) strongly inhibited actin assembly by ForA-C at a molar ratio of $\sim 1: 1$, indicating a strong interaction of the regulatory domains of this DRF (Fig. 2c,d). In dilution-induced depolymerization assays, ForA-C inhibited the depolymerization of actin filaments due to its association with filament barbed ends with a calculated apparent $K_{\mathrm{d}}$ of $4 \mathrm{nM}$ (Fig. 2e,f). Next, we further examined its filament nucleation and elongation activities by single-filament total internal reflection fluorescence (TIRF) microscopy. Nanomolar concentrations of ForA-C noticeably stimulated the nucleation of filaments that subsequently grew with 60 subunits per second, in the presence of profilin I (PFN I), as compared with actin control filaments, which elongated with 18 subunits per second (Fig. 2g-i; Supplementary Movies 5 and 6), illustrating that ForA exerts prototypical formin activity. Collectively, these data therefore supported the notion of ForA contributing to the formation of cortical actin in the trailing edge.

ForA is required for efficient motility in $2 \mathrm{D}$ confinement. To assess the function of ForA in motility, we disrupted the for $A$ gene by homologous recombination in AX2 wild-type cells. Successful elimination was confirmed by PCR and immunoblots using ForA-specific polyclonal antibodies (Fig. 3a). Intriguingly, the elimination of ForA markedly increased the speed of randomly migrating cells in unconfined environments to $11.2 \pm 2.5 \mu \mathrm{m} \mathrm{min}^{-1}$ (mean \pm s.d., $\left.n=126\right)$ as compared with controls with $7.5 \pm 2.1 \mu \mathrm{m} \mathrm{min}^{-1}(n=137) \quad$ (Fig. 3b-d; Supplementary Movie 7). Reconstitution of for $A^{-}$cells with GFP-tagged ForA $\triangle \mathrm{DAD}$ restored motility to wild-type levels, demonstrating the absence of ForA to be the sole cause for the phenotype (Fig. 3c). Notably, however, when compressed under a sheet of agar, for $A^{-}$cells were strongly impaired in $2 \mathrm{D}$-confined 
cell migration $\left(3.0 \pm 1.0 \mu \mathrm{m} \mathrm{min}^{-1}, n=190\right)$ as opposed to wild type $\left(4.3 \pm 1.7 \mu \mathrm{m} \mathrm{min}^{-1}, \quad n=128\right)$ (Fig. 3b,e,f; Supplementary Movie 8). Thus, when comparing migration in unconfined versus 2D-confined settings, the speed of wild-type cells was reduced by
$43 \%$, whereas the speed of for $A^{-}$cells was diminished by $73 \%$. Since myosin-II-based contractility is necessary to allow migration in confined environments (see below), these findings pointed towards a defect associated with the contractile machinery at this point. a

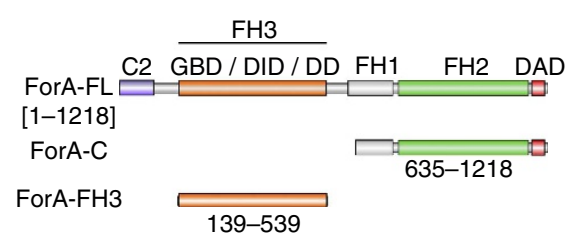

b

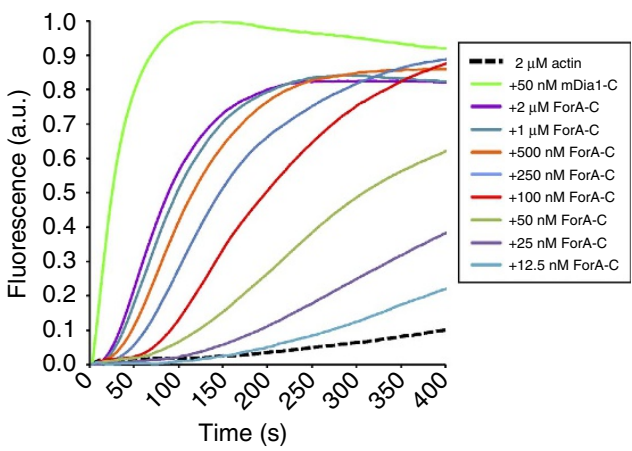

C
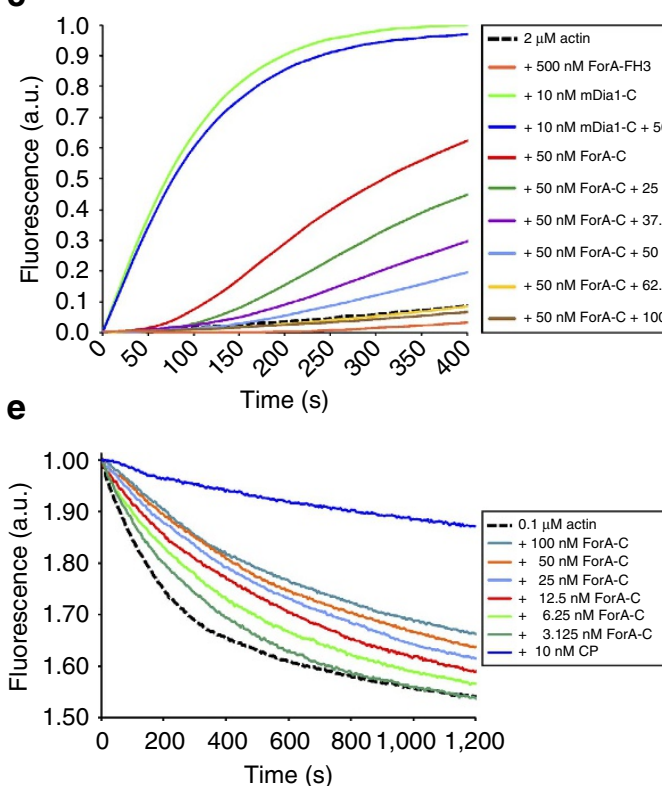

g
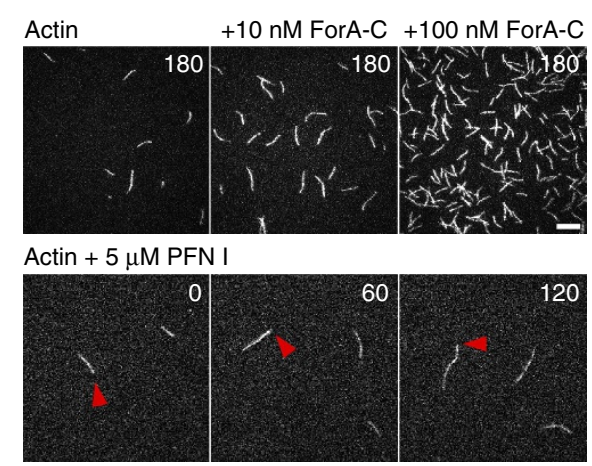

Actin $+5 \mu \mathrm{M}$ PFN I + 10 nM ForA-C

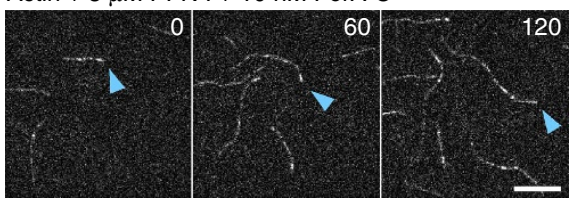

\section{d}

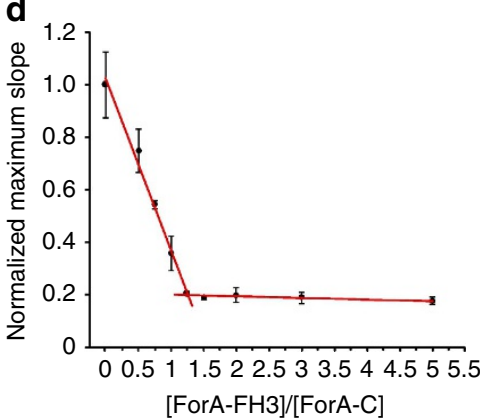

f

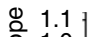

응 1.1

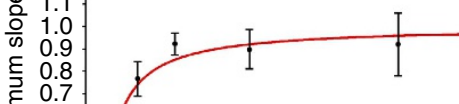

है 0.8

爻 0.6

要 0.4 .

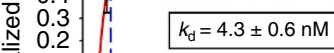

气ू 0.1 .

o

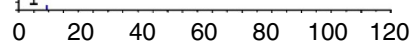

[ForA-C] (nM)

h

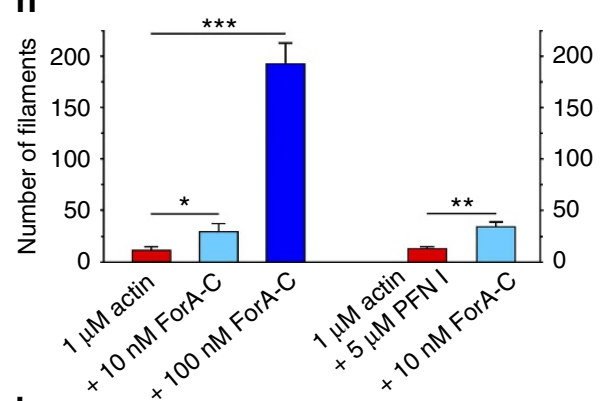

i
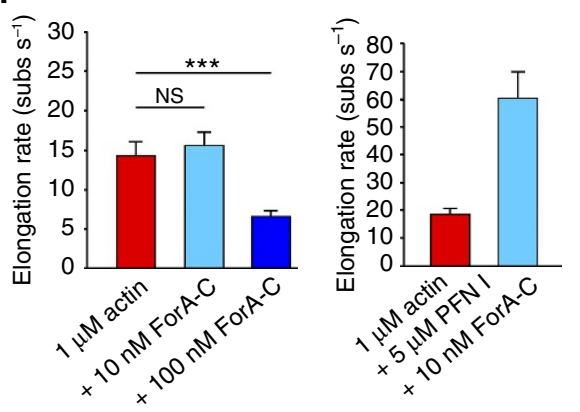
IQGAPs and cortexillin localize like myosin II to the rear. The increased speed of freely migrating for $\mathrm{A}^{-}$cells was reminiscent of the increased motility rates of a Dictyostelium mutant lacking the IQGAP-related protein IQGAP1 (DGAP1) ${ }^{27}$, suggesting a potential mechanistical crosstalk of IQGAP1 and ForA in cell migration. The scaffolding protein IQGAP1 and the closely related IQGAP2 (GAPA) are tightly associated with the heterodimeric actin-bundling protein cortexillin (Ctx), forming preferentially antiparallel bundles in vitro ${ }^{28}$, and notably, all of them are also found in the cleavage furrow of dividing cells ${ }^{29-31}$. We therefore analysed the localization of these proteins fused to GFP in reconstituted mutant backgrounds. Similar to the localization of active ForA and myosin II in polarized, migrating growth-phase cells, both IQGAPs as well as the Ctx isoforms I and II were enriched in the rear cell cortex as opposed to the strong accumulation of F-actin in cell fronts (Fig. 4a). Analysis of cell migration in single and double mutants revealed generally increased motility rates for the iqg $p^{-}$mutants, whereas motility of $c t x^{-}$cells was diminished as compared with the wild type, although the effects of IQGAP2 and CtxII for cell migration in the single mutants were negligible (Fig. $4 \mathrm{~b}$ ).

ForA is required for cortical integrity. The IQGAPs and Ctx were previously shown to be implicated in regulation of cortical tension in adherent cells $^{32,33}$. To assess the role of ForA in cortical integrity in the absence of adhesion forces and compare its contributions with those of the IQGAPs and Ctx in the same experimental set-up, we employed MPA to measure the global mechanical rigidity of freely floating cells, as all of these proteins are evenly distributed in the cortex of unpolarized cells (Fig. 1a; refs 30,31$)$. To preclude secondary responses of the cells to external suction pressure, we quantified the initial projection length $\left(L_{\mathrm{p}, \text { ini }}\right)$ of cells captured from suspension at a constant pressure of $500 \mathrm{~Pa}$ in MPA assays. Loss of ForA, the IQGAPs or of Ctx resulted in significantly longer projection lengths, indicating more unstable cell cortices as compared with the wild type (Fig. 5a,b). Of note, with time only wild-type cells as well as for $\mathrm{A}^{-}$and iqgp $1^{-}$single mutants were able to counteract the suction pressure and actively withdraw from the micropipette, while $16 \%$ of the $c t x I^{-} / c t x I^{-}$and $84 \%$ of the iqgp $1^{-}$/iqgp $2^{-}$ mutants were entirely aspirated (Supplementary Movie 9). This indicated that both double mutants are unable to appropriately reorganize their cortical actin network or suffer from defects in mechanotransduction to counteract the applied force through increased actomyosin-based contractility. We therefore reasoned that the increased motility of for $\mathrm{A}^{-}$cells as well as of iqgp $1^{-}$and iqgp1- /iqgp $2^{-}$mutants could be due to a weakened cell cortex allowing enhanced formation of protrusions in the front. The $c t x I^{-}$and $c t x I^{-} / c t x I I^{-}$mutants though, despite their more fragile cortices, may be slower due to impaired cortex-membrane attachment and filament bundling deteriorating force transmission from the contractile actomyosin system to the plasma membrane at the trailing edge ${ }^{33}$.

ForA synergizes with myosin II in motility. To assess the relevance of myosin-II-based contraction in this context and additionally either confirm or exclude a potential competition between different actin nucleators ${ }^{34}$, we disrupted the for $A$ gene in myosin-II-null cells (Fig. 6a). Analysis of unconfined random cell migration of $m h c A^{-} /$for $A^{-}$-double mutants revealed that loss of ForA no longer enhanced motility in this genetic background (Fig. 6b,c; Supplementary Movie 10), demonstrating that this motility phenotype was strictly dependent on myosin II activity. A quantitative comparison of these mutants in 2D-confined environments was not possible due to the inability of the cells to polarize and migrate under agar in the absence of myosin II (Fig. 6d; Supplementary Movie 11). Despite the fact that $m h A^{-} /$for $A^{-}$-double mutants are immotile, time-lapse imaging and kymograph analyses interestingly revealed that these cells were still able to form small protrusions along their entire periphery, while $m h c A^{-}$cells had an entirely spherical and smooth surface illustrating a complete lack of protrusive activity (Fig. 6d,e; Supplementary Movie 11). These observations are therefore not only consistent with the importance of myosin II in symmetry breaking, but additionally support the inhibitory role of ForA in the formation of cell protrusions, as the protein is evenly distributed in the cell cortex of unpolarized cells (see Fig. 1a; Supplementary Movie 11). Combined, they further demonstrate that under 2D confinement, hydrostatic pressure generated by actomyosin-based contractility must synergize with protrusive activities in the front to drive cell migration.

ForA is required to withstand high actomyosin-based forces. Exposure of Dictyostelium cells to mechanical stress as seen in agar overlay assays is associated with increased myosin II enrichment at the cortex, leading to an augmentation of contraction to counteract the compressive pressure ${ }^{35}$. To directly visualize the contractile machinery in $2 \mathrm{D}$-confined environments, we expressed GFP-tagged myosin II in wild-type and for $A^{-}$cells and analysed their migration by time-lapse confocal imaging under agar. GFP-myosin II was strongly enriched at the rear cortex of migrating wild-type-derived cells (Fig. 7a; Supplementary Movie 12). GFP-myosin II localization in for ${ }^{-}$-derived cells was similar, however, and as opposed to control, time-lapse imaging interestingly revealed that these cells

Figure 2 | ForA is an autoinhibited formin that promotes actin assembly. (a) ForA constructs used for biochemical analyses. C2, C2 domain; GBD, GTPase-binding domain; DID, diaphanous inhibitory domain; DD, dimerization domain; FH, formin homology domain; DAD, diaphanous autoinhibitory domain. (b) ForA-C promotes actin assembly in pyrene assays in a concentration-dependent manner. A total of $50 \mathrm{nM}$ mDial-C served as a positive control. (c) ForA is autoinhibited. ForA-FH3 specifically inhibited actin polymerization of ForA-C in a concentration-dependent manner, but had no effect on mDia1-C. (d) Maximum slopes of the polymerization curves shown in c revealed high affinity of ForA-FH3 to ForA-C. Intersection of the solid lines (red) marks the molar ratio required for full inhibition indicating 1:1 stoichiometry. Bars represent mean \pm s.d. $n=4$. (e) ForA-C inhibits actin disassembly in dilution-induced depolymerization assays, demonstrating interaction with filament barbed ends. $10 \mathrm{nM}$ capping protein (CP) served as a control for highaffinity binding to filament barbed ends. (f) Maximum slopes of the depolymerization curves as shown in e were fitted assuming one binding site at filament barbed ends and revealed an apparent $K_{d}$ of $4.3 \pm 0.6 \mathrm{nM}$. Error bars represent mean \pm s.d., $n=4$. (g) Time-lapse micrographs of actin assembly visualized by in vitro TIRF microscopy. Upper panel displays number of nucleated filaments in the absence or presence of 10 or $100 \mathrm{nM}$ ForA-C. Lower two panels illustrate barbed ends growth of a control filament (red arrowhead) and a ForA-C elongated filament (blue arrowhead) in presence of PFN I. Time is in seconds. Scale bars, $10 \mu \mathrm{m}$. (h) Quantification of nucleated filaments obtained from TIRF microscopy experiments as shown in $\mathbf{g}$. Bars represent mean \pm s.d., $n=3$. ${ }^{\star \star}{ }^{\star} P \leq 0.001,{ }^{\star \star} P \leq 0.01,{ }^{\star} P \leq 0.05$ ( $t$-test). (i) In the absence of PFN I, addition of $100 \mathrm{nM}$ ForA-C (dark blue) led to significantly reduced elongation rates as compared with control (red). In the presence of PFN I, ForA-C-associated filaments (light blue) grew threefold faster than control filaments. Bars represent mean \pm s.d., $n>20$. ${ }^{\star \star \star} P \leq 0.001$, NS, not significant (Mann-Whitney U-test) 
a

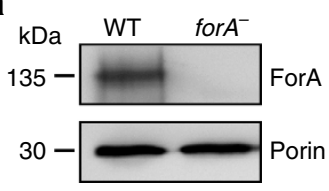

C

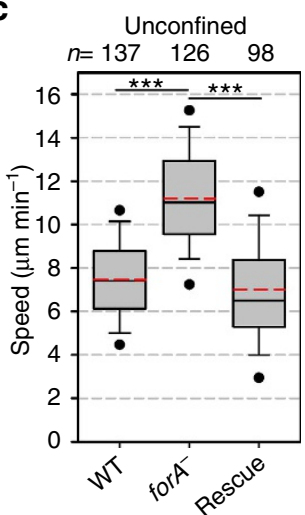

e

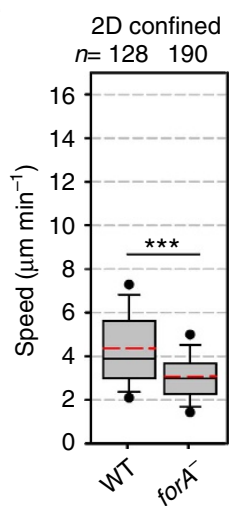

b

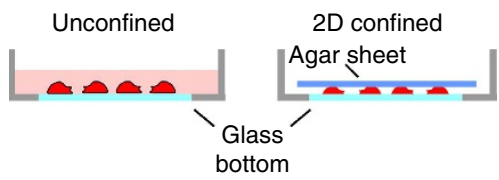

d

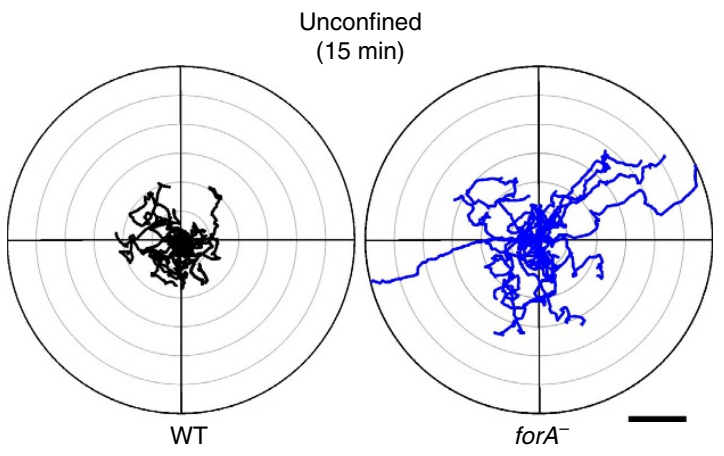

f

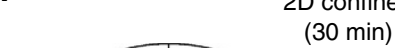

Figure 3 | ForA effects motility in unconfined and 2D-confined environments. (a) Elimination of ForA was confirmed by immunoblotting. Porin was used as a loading control. (b) Scheme for analyses of cell migration in unconfined and 2D-confined settings. (c) Quantitative analysis of random cell

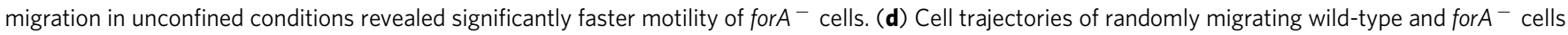
of representative samples in radar plots illustrate faster motility of the mutant cells as compared with wild type (see Supplementary Movie 7).

(e) Quantitative analysis of random cell migration in 2D-confined environments. When sandwiched between the glass surface and a thin sheet of agar, for $\mathrm{A}^{-}$mutants showed diminished migration rates as compared with wild type (see Supplementary Movie 8). (f) Cell trajectories of randomly migrating wild-type and for $A^{-}$cells (20 each) of representative samples in radar plots illustrate compromised motility of for $A^{-}$cells in confinement (see Supplementary Movie 8). Boxes in $\mathbf{c}$ and $\mathbf{e}$ indicate $25-75$ percentiles, the whiskers mark the $10^{\text {th }}$ and $90^{\text {th }}$ and the outliers the $5^{\text {th }}$ and $95^{\text {th }}$ percentile. Red dashed lines indicate mean values. $n$, number of tracked cells. ${ }^{\star \star}{ }^{\star} P \leq 0.001$ (Mann-Whitney $U$-test). Scale bars, $40 \mu \mathrm{m}$ (d,f).

repeatedly formed extensive blebs in their rear (Fig. 7b; Supplementary Movie 13). Blebs form due to detachment of the plasma membrane from a weakened or ruptured cortex by hydrostatic pressure generated by myosin-IIdriven contraction ${ }^{36}$. Quantitative analyses showed a 13-fold higher occurrence $\left(0.91 \pm 0.27 \mathrm{~s}^{-1}\right.$, mean \pm s.d., $n=17$ versus $0.07 \pm 0.07 \mathrm{~s}^{-1}, n=23$ ) and an about a fourfold larger size $\left(19.66 \pm 7.89 \mu \mathrm{m}^{2}\right.$, mean \pm s.d., $n=38$ versus $5.66 \pm 4.66 \mu \mathrm{m}^{2}$, $n=31$ ) of these rearward blebs in for $A^{-}$cells as compared with control (Fig. 7c,e). Strikingly, the determination of centroid velocity and rear bleb size revealed a periodic and inverse correlation between cell speed and blebbing activity in the mutant, as periods with bleb growth accurately matched phases with decreased cell speed (Fig. 7d; Supplementary Movie 14). ForA is obviously not required for myosin-II-driven contraction per se, as evidenced by the excessive blebbing in GFP-myosin-IIexpressing for $A^{-}$cells, but is a critical component to prevent posterior blebbing. Fluorescence labelling of fixed cells further revealed a substantially increased accumulation of F-actin in the rear of compressed cells as opposed to freely moving cells (Fig. 7f). Hence, in confined environments the cortex is strengthened by relocation of actin, myosin II and other actin-binding proteins such as the Ctx/IQGAP1 complex (Supplementary Fig. 3) to the rear. However, in spite of these adaptations the posterior cortical actin meshwork in the formin mutant was evidently still not able to provide the mechanical strength of the cortex required for efficient cell migration in 2Dconfined environments. Thus, ForA serves to protect the cell against unproductive blebbing upon the increased contractile forces in response to mechanical stress by generating a specialized subset of actin filaments as the foundation of the protective actin sheath in the rear cortex to drive motility.

Recruitment of ForA to the cell rear. Next, we asked how ForA is positioned in the trailing edge of cells. The protein contains a 


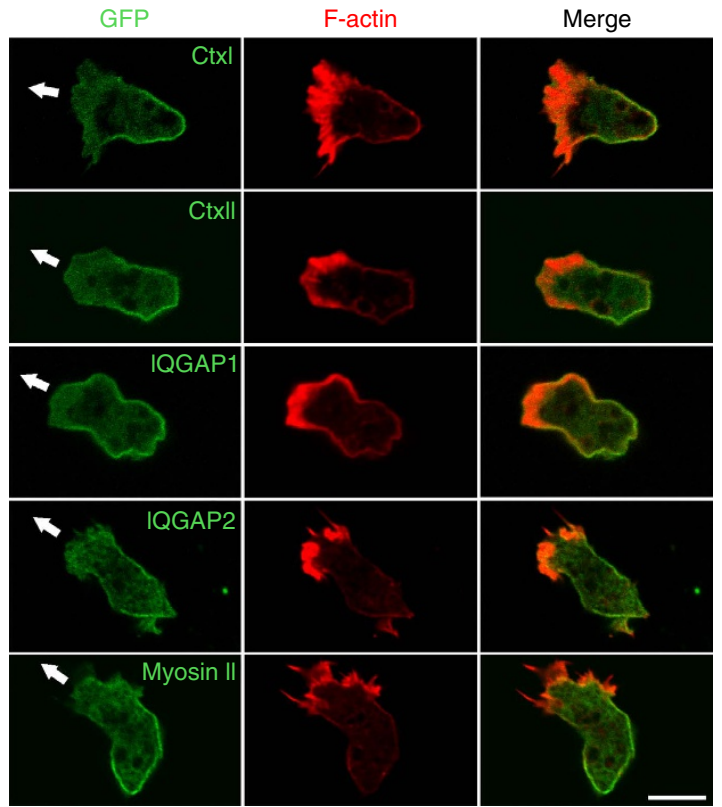

b

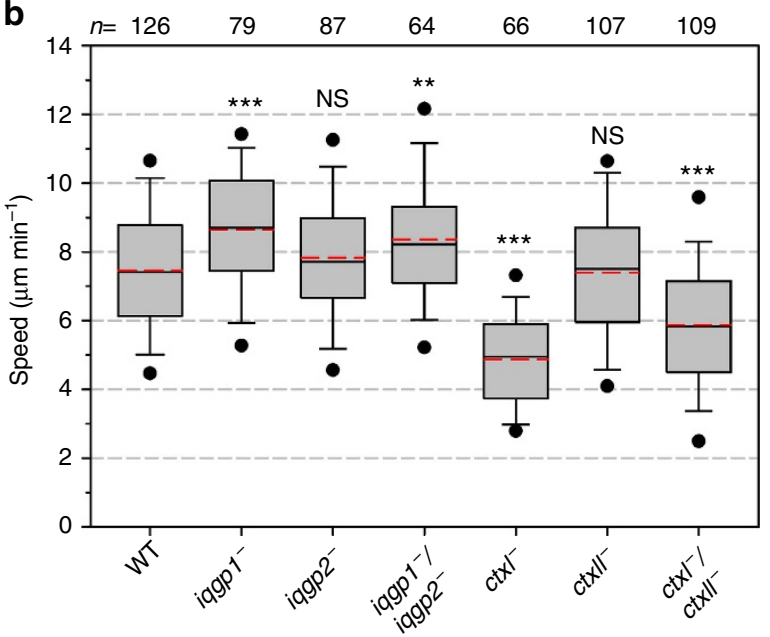

Figure 4 | Ctx and the IQGAPs localize like myosin II to the rear and affect cell migration. (a) The proteins indicated were expressed as GFP fusions in the respective knockout mutants, fixed and labelled with Atto488-conjugated nanobodies (green) to enhance the GFP signal. Filamentous actin was stained with rhodamine phalloidin (red). The white arrow marks the direction of cell movement. Scale bar, $10 \mu \mathrm{m}$.

(b) Quantitative analysis of random cell motility of iqgp ${ }^{-}$and $c t x^{-}$mutants in unconfined environments. $n$, number of tracked cells. ${ }^{\star \star \star} P \leq 0.001$;

${ }^{\star \star} P \leq 0.01 ; N S$, not significant (Mann-Whitney $U$-test). Statistical differences refer to wild type.

putative C2 domain at its $\mathrm{N}$ terminus (aa 1-100), suggesting electrostatic interactions with acidic phospholipids ${ }^{37}$. To directly test interaction of this region with lipids in vitro, purified GSTtagged ForA-C2 was assessed by co-flotation assays with liposomes containing various phosphoinositides. ForA-C2 bound preferentially to phosphatidyl-inositol-(4,5)-bisphosphate $\left(\mathrm{PI}(4,5) \mathrm{P}_{2}\right)$ (Fig. 8a), which accumulates in the trailing edge of migrating cells ${ }^{10}$. To directly test whether the C2 domain is critical for targeting in vivo, we analysed the localization of the $\mathrm{N}$-terminal half of ForA containing the $\mathrm{C} 2$ domain (ForA-N), the active formin lacking $\mathrm{C} 2$ (ForA $\Delta \mathrm{C} 2 \Delta \mathrm{DAD}$ ) as well as the $\mathrm{C} 2$ domain alone (ForA-C2) in for $A^{-}$cells. While GFP-tagged ForA-N localized to the cortex in the rear like the active formin,

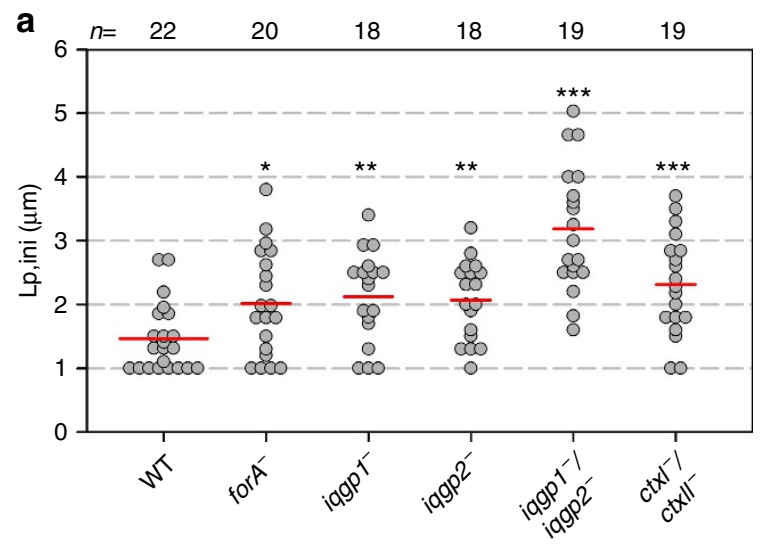

b

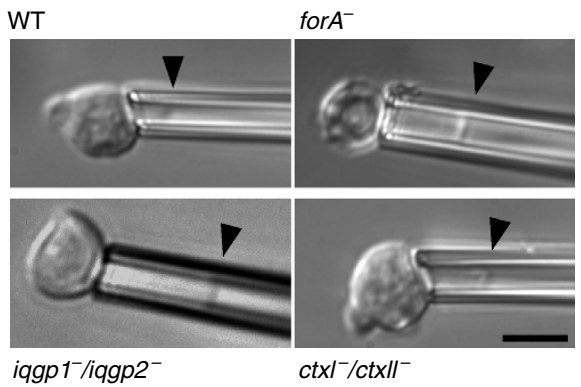

Figure 5 | ForA is required for cortical integrity. (a) Initial projection length $L_{p, \text { ini }}$ of wild-type and mutant cells determined by MPA using a constant suction pressure of $500 \mathrm{~Pa}$. Red lines, mean values. $n$, number of analysed cells. ${ }^{\star \star \star} P \leq 0.001 ;{ }^{\star \star} P \leq 0.01 ;{ }^{\star} P \leq 0.05$ (Mann-Whitney $U$-test). Statistical differences refer to wild type. (b) Development of projections (black arrowheads mark menisci) after $145 \mathrm{~s}$ in representative samples. Scale bar, $10 \mu \mathrm{m}$.

both ForA $\Delta \mathrm{C} 2 \Delta \mathrm{DAD}$ and ForA-C2 were evenly distributed in the entire cytoplasm (Fig. 8b), demonstrating that ForA-C2 interaction with $\mathrm{PI}(4,5) \mathrm{P}_{2}$ is essential but not sufficient for targeting to the cell rear. Thus, additional signals seem to be mandatory for subcellular localization. Obviously, ForA targeting does not require actin, since the appropriately localizing ForA-N lacks all actin interaction surfaces. Since CtxI was previously also shown to interact with $\mathrm{PI}(4,5) \mathrm{P}_{2}$ (ref. 38), the Ctx/IQGAP complex may be recruited in a similar fashion and mediate the crosslinking of ForA-generated actin filaments beneath the plasma membrane.

IQGAPs sequester active Racl in the rear. We then addressed the somewhat unexpected localization of the Racl-binding protein IQGAP1 in the rear ${ }^{39}$, because Rac1 is well established and mandatory to induce migratory protrusions downstream of Scar/ WAVE signalling in Arp2/3 complex-mediated actin assembly in the front ${ }^{40}$. Consistently, overexpression of wild-type Rac1 leads to increased Rac1-GTP levels and augments motility of Dictyostelium cells ${ }^{27}$. We noticed, however, that wild-type Rac1 overexpressed at similar levels in the faster iqgp1 ${ }^{-}$mutants did not further increase cell speed (Fig. 8c; Supplementary Fig. 4). Since both IQGAPs interact exclusively with active Rac1 (refs 30,41), we reasoned that presumably due to elevated levels of accessible Rac1-GTP caused by absence of the Racl effector IQGAP1, the Arp2/3 complex might be already fully activated in iqgp $1^{-}$mutants to drive actin assembly in the leading edge. In line with this, the fast iqgp1 ${ }^{-}$mutants were previously reported 
a

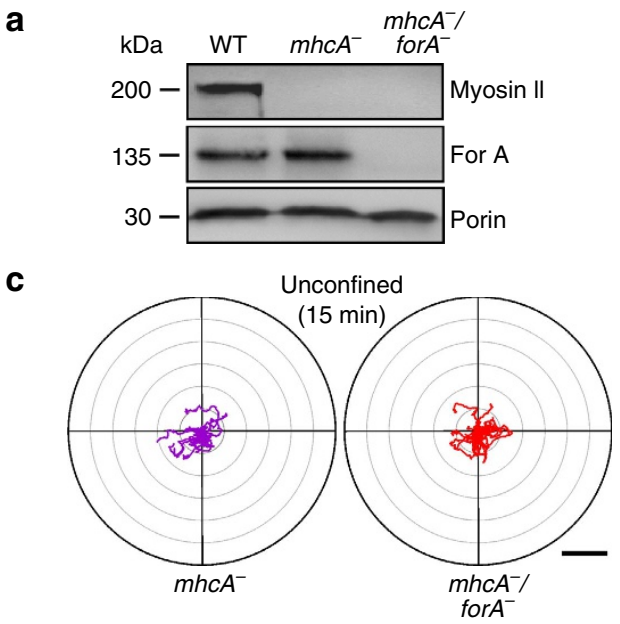

d $m h c A^{-}$

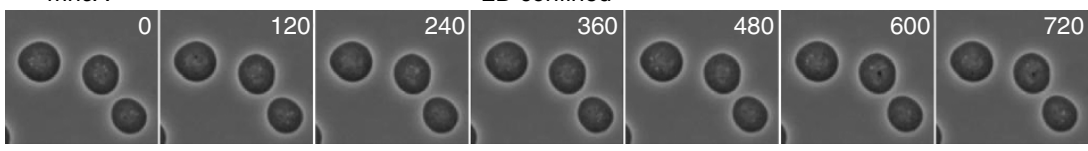

$m h c A^{-} /$for $A^{-}$

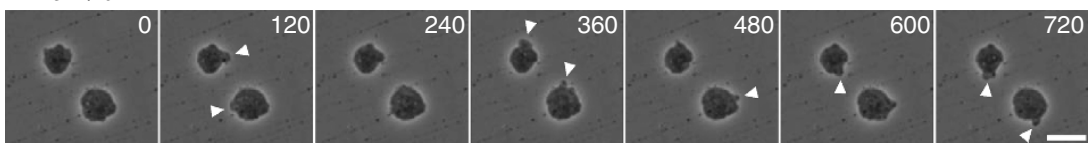

e $m h c A^{-}$
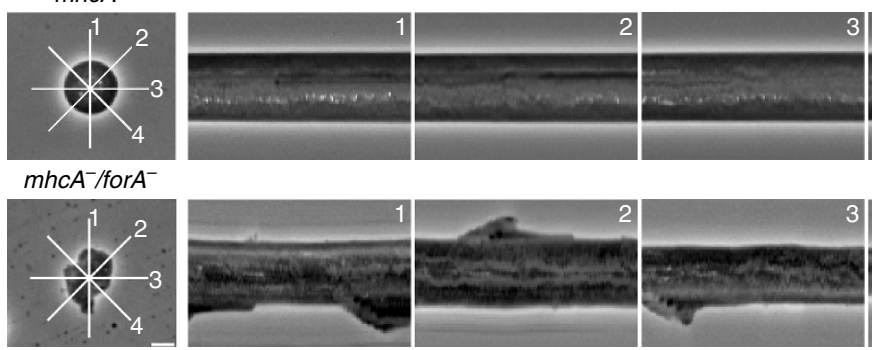

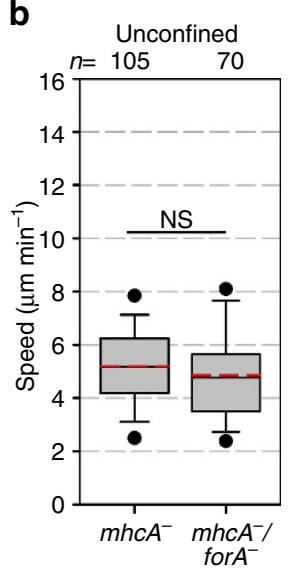

Figure 6 | The ForA motility phenotypes are myosin II dependent. (a) Elimination of ForA in mhcA- cells was confirmed by immunoblot using myosin-IIspecific antibodies. Porin served as a loading control. (b) Quantitative comparison of random cell migration of $m h c A^{-}$and $m h c A^{-} /$for $A^{-}$cells in unconfined environments revealed no statistical difference. $n$, number of tracked cells. NS, not significant (Mann-Whitney $U$-test). (c) Radar plots of 20 cell trajectories each taken from representative samples. Scale bar, $40 \mu \mathrm{m}$. (d) Still images from time-lapse phase-contrast movies refer to Supplementary Movie 8 and illustrate that both cell lines are unable to migrate under agar. However, only $m h c^{-} /$for $A^{-}$cells were able to form protrusion along their periphery (white arrowheads) in 2D confinement. Scale bar, $20 \mu \mathrm{m}$. (e) Kymograph analyses of $m h c A^{-}$and $m h c A^{-} / f o r A^{-}$cells, respectively, that were selected from the movies depicted in d. Kymographs were generated along the indicated lines. Horizontal scale bar, $100 \mathrm{~s}$. Vertical scale bar, $10 \mu \mathrm{m}$.

to generate excessive cell protrusions, while the overexpression of IQGAP1 led to round cells largely devoid of pseudopods and filopodia $^{42}$. To test our hypothesis, we performed GTPase activation assays using maltose-binding protein (MBP)-tagged PAK1-GBD and indeed found higher levels of active Racl in the iqgp ${ }^{-}$mutants (Fig. 8d). Thus, in addition to their effects on cortical integrity, the IQGAPs seem to suppress activation of residual amounts of Arp $2 / 3$ complex in the rear by sequestration of active Rac1.

ForA is the functional homologue of vertebrate mDial. Since actomyosin-based contraction is exploited by many cell types to drive or support motility, we finally asked whether higher eukaryotes could employ a related mechanism to generate a rigid cortex in the rear. Although vertebrates do not express Ctx, human IQGAP1 by itself might replace its function, since it was previously reported to crosslink actin filaments in $v i t r 0^{43}$, and have shown to accumulate in the trailing edge, at least in B16-F10 and WM239A melanoma cells ${ }^{44,45}$. To corroborate these findings in a commonly used and highly migratory cell line, we transfected B16-F1 mouse melanoma cells with enhanced (E)GFP-tagged IQGAP1 (ref. 46) and monitored its subcellular localization. In moderately expressing cells IQGAP1 localized rather weakly to actin-based structures in the front, but was substantially enriched in the trailing edge (Fig. 9a). Since contractility in vertebrates is regulated by Rho signalling to mDia1 and ROCK to trigger actin polymerization and activate myosin ${ }^{47}$, we additionally monitored localization of constitutively active EGFP-tagged mDial (EGFP$\mathrm{mDia} 1 \triangle \mathrm{DAD})$. Strong overexpression of active mDial in B16-F1 cells induced aberrant cell morphologies and led to massive disorganization of the F-actin cytoskeleton. By contrast, in moderately expressing cells, active mDial was excluded from the leading edge, but profoundly localized like ForA to the cortex 
a

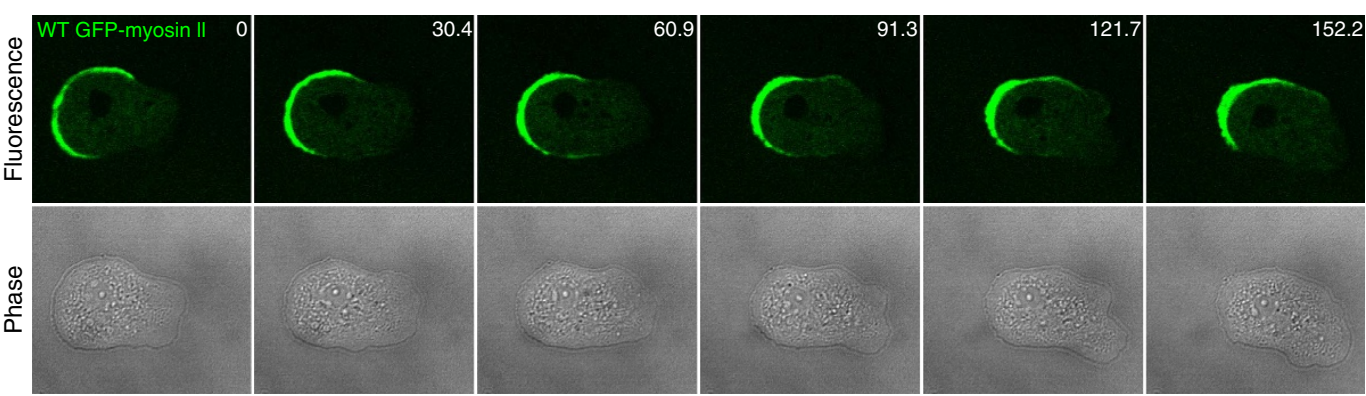

b

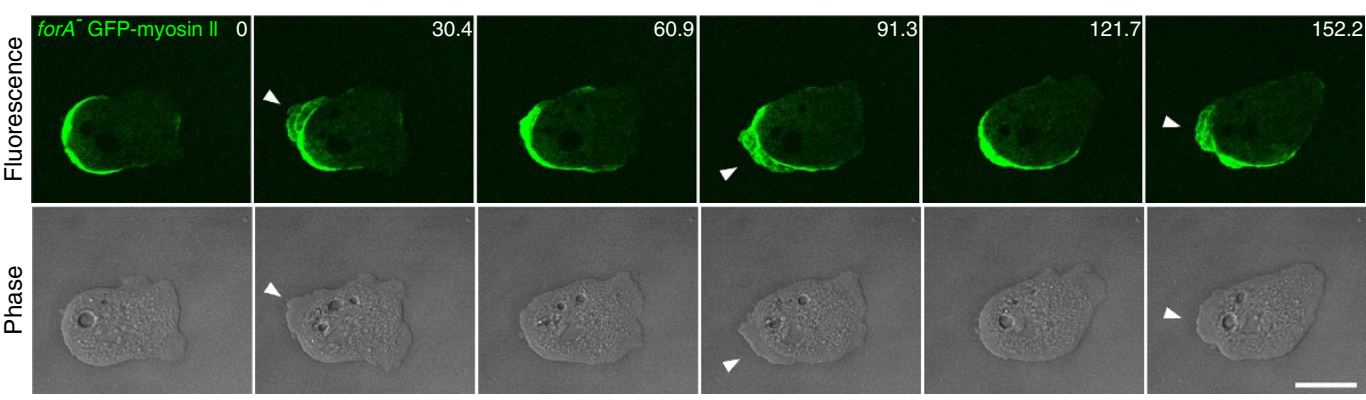

C

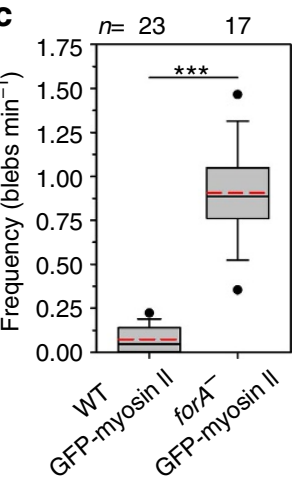

d

e
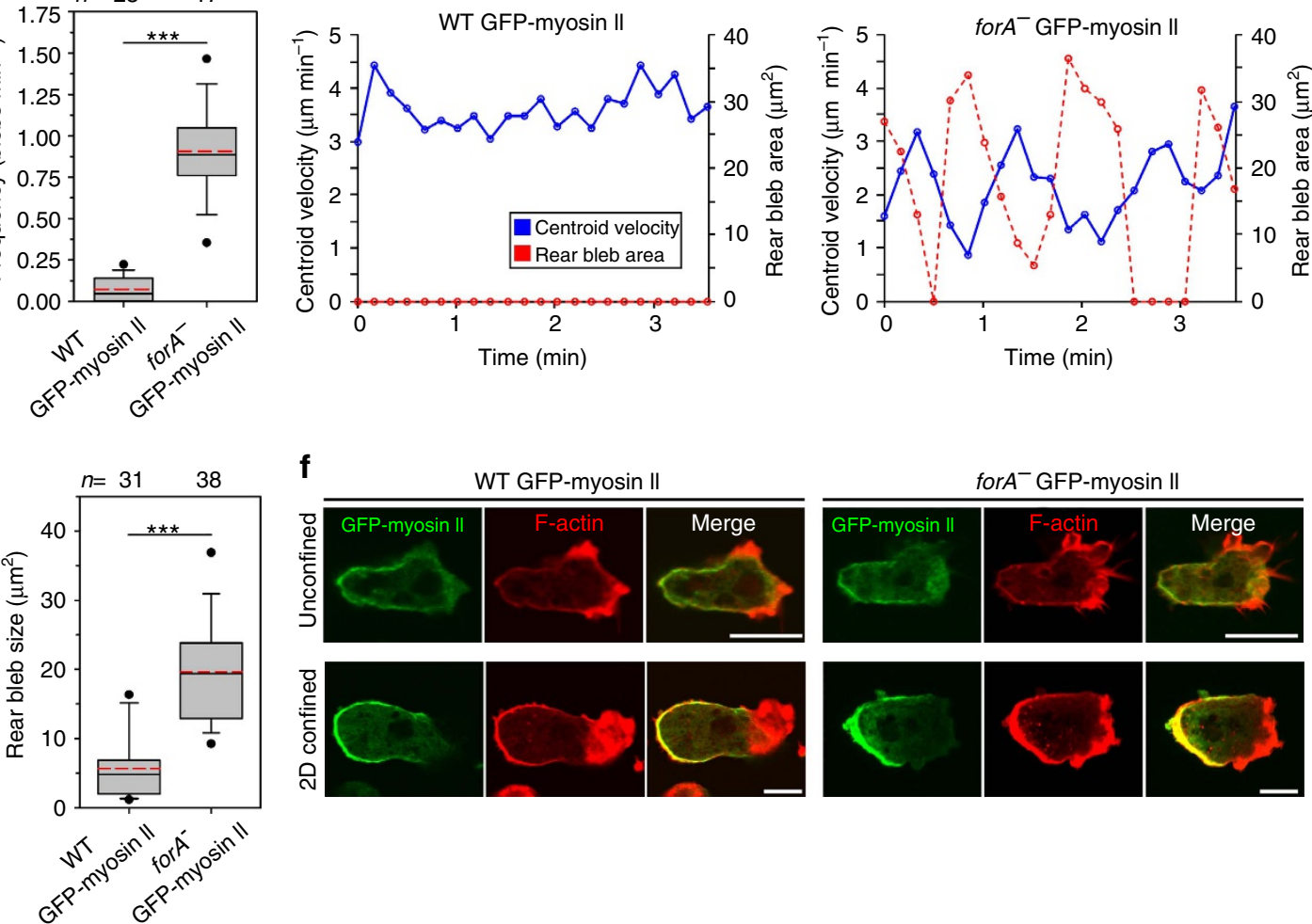

f
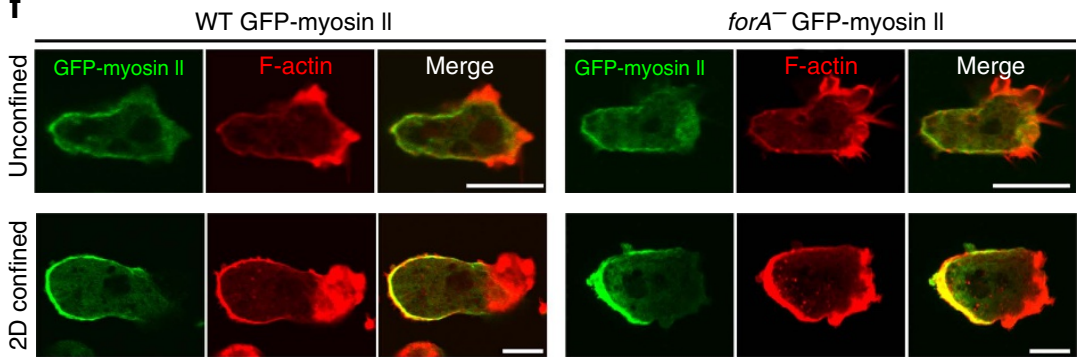

Figure 7 | ForA prevents blebbing at the rear during cell migration in 2D-confined environments. (a,b) Accumulation of GFP-myosin II in the rear of wild-type and for $A^{-}$cells migrating under agar. Arrowheads indicate posterior blebs formed in for $A^{-}$cells. Images refer to Supplementary Movies 12 and 13. Scale bar, $10 \mu \mathrm{m}$. (c,e) Frequency and size of posterior blebs in wild-type and for $A^{-}$cells expressing GFP-myosin II under agar. $n$, number of analysed blebs. ${ }^{\star \star *} P \leq 0.001$ (Mann-Whitney U-test). (d) Inverse correlation of cell motility and rear blebbing. Centroid velocities (blue solid line) and bleb areas (red dashed line) of representative frames shown in $\mathbf{a}$ and $\mathbf{b}$. Excessive blebbing of for $\mathrm{A}^{-}$cells resulted in diminished migration speed. (f) Increased accumulation of myosin II and filamentous actin in the rear after compression under agar. After fixing the cells, GFP-tagged myosin II was enhanced with Atto488-conjugated nanobodies (green). Filamentous actin was stained with rhodamine phalloidin (red). Scale bars, $5 \mu \mathrm{m}$.

of the trailing edge together with cortical F-actin and myosin IIA (Fig. 9b; Supplementary Movie 15). Active mDial also relocated to the new prospective rear in repolarizing cells and vanished before the formation of migratory protrusions (Fig. 9c).
These findings collectively suggest that Dictyostelium ForA represents the functional homologue of mDial pointing towards a conserved mechanism in the generation of the rear cortex across species. 
a

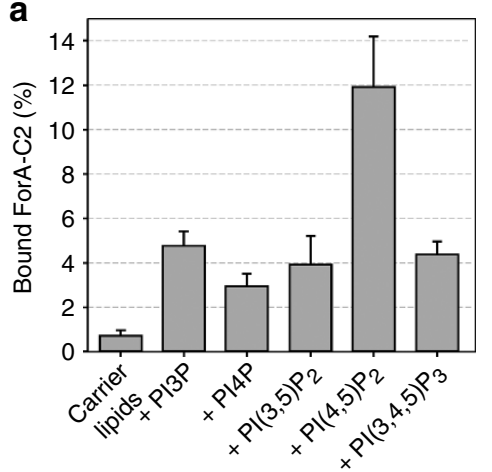

b

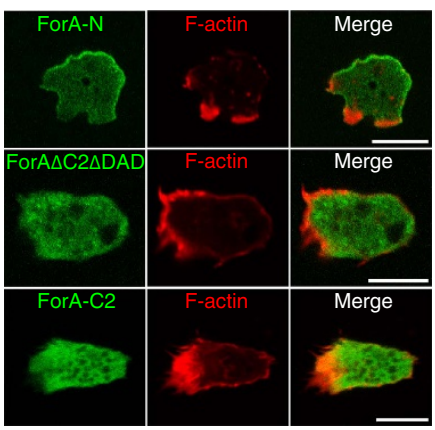

C

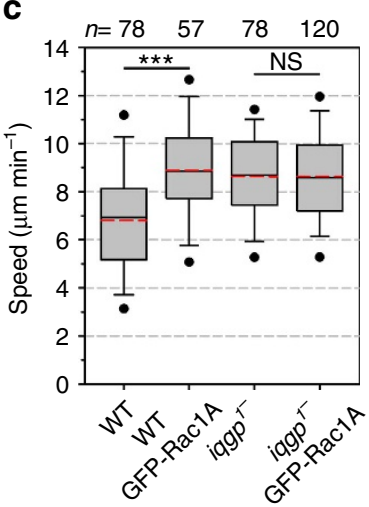

d

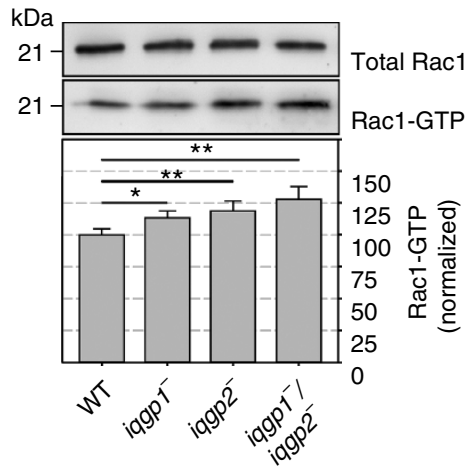

e

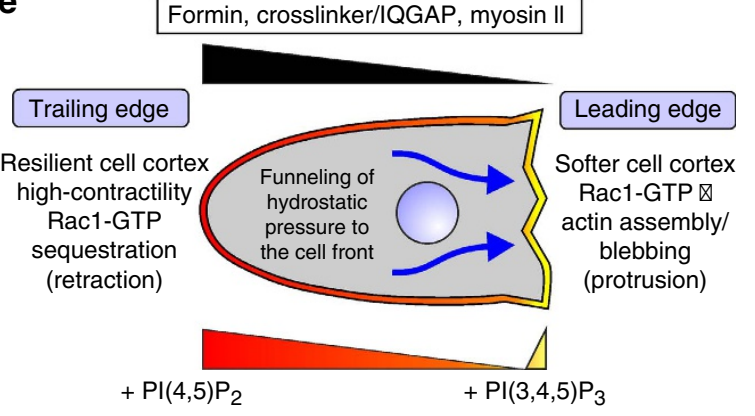

Figure 8 | Regulatory mechanisms establishing cortical rigidity in the rear. (a) The For A- $\mathrm{C} 2$ domain binds preferentially to $\mathrm{PI}(4,5) \mathrm{P}_{2}$ in vitro. Quantification of ForA-C2 binding to phosphoinositide-containing liposomes. Data are mean \pm s.d., $n=4$. (b) ForA-C2 is essential for subcellular targeting. After reconstitution in for $\mathrm{A}^{-}$cells with truncated ForA constructs fused to GFP, the cells were fixed and labelled with Alexa488-conjugated nanobodies (green) and rhodamine phalloidin to visualize filamentous actin (red). Deletion of C2 abolished trailing edge localization, but C2 alone was not sufficient for targeting to the rear. Scale bar, $10 \mu \mathrm{m}$. (c) Ectopic expression of GFP-tagged Rac1A increases random motility in wild-type cells, but not in iqgp1- cells. $n$, number of analysed cells. ${ }^{\star \star \star} P \leq 0.001$; NS, not significant (Mann-Whitney U-test). (d) MBP-PAK1-GBD pull-downs showed increased levels of active Rac1 in single- and double-iqgp ${ }^{-}$mutants. Error bars represent mean \pm s.d., $n=4$. ${ }^{\star} P \leq 0.05 ;{ }^{\star} P \leq 0.01$ (two-tailed, unpaired Student's $t$-test). (e) Model $\mathrm{PI}(3,4,5) \mathrm{P}_{3}$ signalling guides protrusion to the front ${ }^{10}$, whereas $\mathrm{PI}(4,5) \mathrm{P}_{2}$ positions the resilient cortical meshwork together with contraction machinery to the rear. For more details see text.

\section{Discussion}

Amoeboid cell migration is context dependent and governed by the balanced interplay of cell-substrate adhesion, together with the cortical activities eliciting protrusion and contraction ${ }^{5}$. The results reported here provide new insights into the molecular mechanisms involved in cortex formation, mechanics and its function during migration of Dictyostelium cells in unconfined and 2D-confined environments. On the basis of the data, we propose that ForA initiates the formation of linear filaments beneath the plasma membrane that are mechanically reinforced by the antiparallel actin crosslinker Ctx and IQGAP-related proteins to generate a specialized actin meshwork in the rear. This resilient compartment is necessary to withstand the amplified actomyosin-based contractile forces in 2D-confined environments and to drive motility. Our hypothesis, diagrammed in Fig. 8e, suggests that in wild-type cells internal hydrostatic pressure generated by myosin-II-based contraction is efficiently gated by the robust cortex in the rear to the softer front to promote cell protrusion. Consistently, similar to $c t x^{-}$and iqgp ${ }^{-}$ mutants, for $A^{-}$cells show substantial defects in cortical rigidity as evidenced by the aspiration assays. Moreover, the impaired migration of for ${ }^{-}$cells under agar was associated with excessive blebbing in regions with highest myosin II accumulation. This illustrates not only the mechanical weakness of the rear cortex but also the ability of the cells to contract in the absence of the formin in spite of a generally increased enrichment of actin, myosin II, Ctx and IQGAP to the back (Fig. 7f; Supplementary Fig. 3). These findings therefore clearly argue against instantaneous pressure equilibration in the cytosol and instead support the concept of non-equilibration of heterogeneous transients in the pressure field of blebbing cells ${ }^{48}$. Interestingly, the migration of MDA-MB231 human breast adenocarcinoma cells in three-dimensional Matrigel was previously reported to be exclusively driven by blebbing in the trailing edge ${ }^{49}$. How can these apparently contradictory findings be reconciled? We hypothesize that in three-dimensional Matrigel, rear blebs of MDA-MB-321 cells could provide repulsive forces in the rear by pushing against the adjacent collagen fibres of the matrix to move the cells forward, while in Dictyostelium even the extensive posterior blebs formed under agar cannot exert repulsive forces against the surrounding liquid due to its low mechanical resistance.

Myosin-II-null cells are motile in unconfined conditions, albeit they migrate with only half the speed of wild-type cells ${ }^{24}$. Subsequent work revealed two mechanically distinct modes of amoeboid movement characterized by the formation of two different cell-surface protrusions, namely actin-rich pseudopods or blebs ${ }^{50}$. The hydrostatic pressure-driven blebs are strongly impaired by perturbation of myosin-II-based contractility or changes in external osmolarity that affects internal hydrostatic pressure. Our analyses of mutants lacking myosin II and ForA in different combinations allowed us to expand on these findings and assess the interplay and contribution of these proteins for motility in unconfined and 2D-confined scenarios. Intriguingly, we observed an almost doubled migration speed of for $A^{-}$cells as compared with wild-type cells in unrestricted conditions. This enhanced motility phenotype is dependent on myosin II function 
a

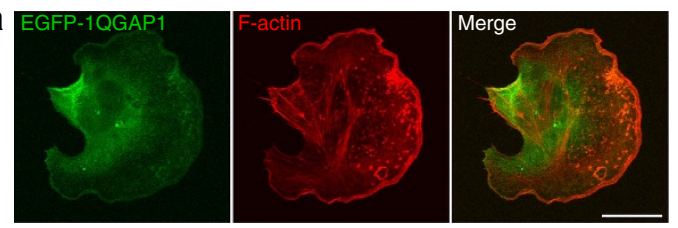

b
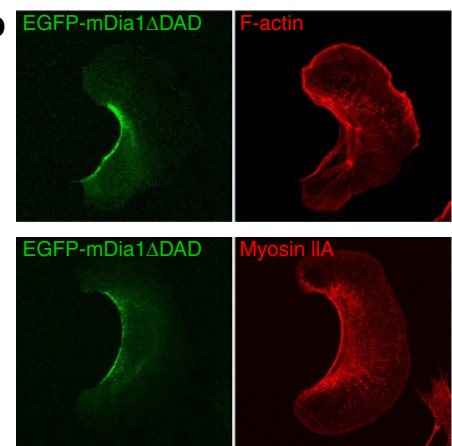
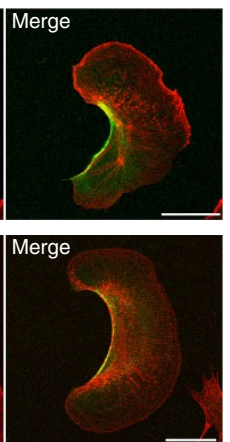

C

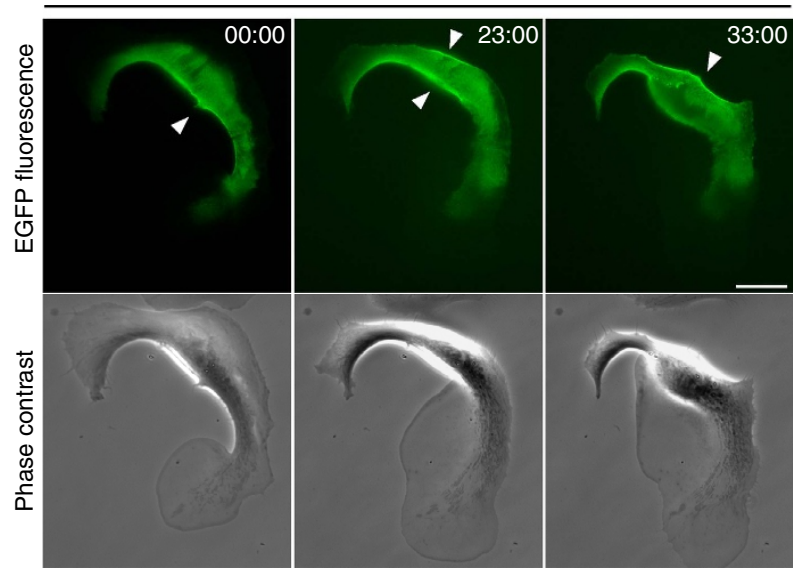

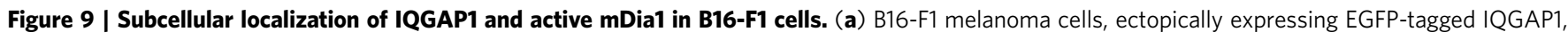
were plated on laminin-coated glass coverslips, fixed and labelled with Alexa488-conjugated nanobodies (green) and rhodamine phalloidin to visualize filamentous actin (red). Note the enrichment of IQGAP1 in the rear. (b) Subcellular localization of ectopically expressed EGFP-tagged active mDia1 in B16-F1 cells migrating on laminin. The cells were processed as described in a, but were additionally stained for myosin IIA. Active mDia1 was excluded from the leading edge and other actin-rich structures, with the exception of its prominent cortex localization in the trailing edge, where it colocalized together with cortical F-actin and myosin IIA. (c) mDia1 dynamics in repolarizing cells. Relocalization of active mDia1 in repolarizing B16-F1 cells to the new prospective end is indicated by white arrowheads and comparable to active ForA in Dictyostelium cells. Still images from time-lapse movies are shown. Time corresponds to min:s. Scale bars, $20 \mu \mathrm{m}$.

(Fig. 6b,c), demonstrating involvement of actomyosin contraction associated with generation of hydrostatic pressure in this process. Given the less rigid cortical actin meshwork in for $A^{-}$cells and provided that the strength of actomyosin-generated forces and the resulting pressure in wild-type and for $A^{-}$cells are comparable, we assume that the increased speed of the mutant is caused by increased compliance of the cortex allowing easier compression of the cells in the back and easier deformability in their front. In 2D confinement, however, augmented hydrostatic pressure evidently exceeds a tolerable limit that disrupts the rear cortex leading to unproductive blebbing that diminishes motility of these cells. Moreover, we found that $\mathrm{MhcA}^{-}$cells are unable to migrate under agar (Fig. 6d), which emphasizes the importance of myosin-II-based contractile forces and generation of hydrostatic pressure for motility of Dictyostelium cells in $2 \mathrm{D}$ confinement. Although the $m h c A^{-} /$for $A^{-}$cells were also immotile under agar, in contrast to $m h c A^{-}$cells, they still formed small protrusions at their periphery. We therefore conclude that in $2 \mathrm{D}$ confinement, hydrostatic pressure generated in the rear must synergize with protrusive activities in the leading edge to allow cell migration.

Efficient cell migration not only requires protrusion in the front and contraction in the back, but also entails inhibition of lateral and rear protrusions. This function appears also to be ascertained by the ForA-based cortical meshwork as we were unable to detect the formation of any protrusion from cortical regions with high ForA accumulation. In case protrusions were spontaneously formed in the rear, these events were invariably preceded by disappearance of the formin (Fig. 1b). This notion is consistent with the lack of protrusions in unpolarized $\mathrm{mhcA}^{-}$ cells under agar, which still contain ForA evenly distributed in their cell cortex, and was further substantiated by the dynamic redistribution of ForA in repolarizing cells (Fig. 1c,d). Consistent with our data and previous work ${ }^{51}$, we further propose that inhibition of lateral protrusion in migrating cells is additionally sustained by the IQGAPs through sequestration of active Racl to prevent undesirable activation of the Arp $2 / 3$ complex.
In vertebrates, cortical actin flow and myosin II have been proposed to localize components of the contractile machinery to the rear ${ }^{52,53}$. However, in migrating Dictyostelium cells, F-actin was reported to move rearward with a much slower velocity than the movement of the centroid ${ }^{54}$. Thus, it is unlikely that actin flow directly controls cell migration in these cells. The distribution of ForA is also myosin II independent, as the formin is still actively recruited to the rear in myosin-II-null cells (Supplementary Fig. 5). We instead found that ForA localization is regulated by $\mathrm{PI}(4,5) \mathrm{P}_{2}$ that is enriched in the trailing edge of polarized cells ${ }^{10}$. Thus, the previously reported defect of mutants lacking PTEN (phosphatase tensin homologue)-which is the main enzyme converting phosphatidyl-inositol- $(3,4,5)$ triphosphate $\left(\mathrm{PI}(3,4,5) \mathrm{P}_{3}\right)$ back to $\mathrm{PI}(4,5) \mathrm{P}_{2}$-in the inhibition of lateral protrusions may be caused by the inability of ForA to localize in the absence of a perturbed $\mathrm{PI}(4,5) \mathrm{P}_{2}$ differential $^{55}$. By the use of truncated ForA constructs, we show that the $\mathrm{PI}(4,5) \mathrm{P}_{2}$ binding C2 domain is essential, although alone, it is not sufficient for targeting to the rear. Since the accurately targeting N-terminal fragment not only encompasses the $\mathrm{C} 2$ domain, but also contains a GTPase-binding site, targeting to the rear most likely requires the concurrent interaction of both binding sites with $\mathrm{PI}(4,5) \mathrm{P}_{2}$ and $\mathrm{a}$ as yet unknown activated GTPase. Consistently, mDial lacking either its $\mathrm{PI}(4,5) \mathrm{P}_{2}$-interacting $\mathrm{N}$-terminal basic region or its GBD fail to localize to the membrane $e^{56,57}$

Finally, we provide evidence that related mechanisms could also be utilized by higher eukaryotes. Although vertebrates do not express Ctx, we propose that human IQGAP1 might already substitute its function. Consistent with previous work ${ }^{45,58}$, we show that IQGAP1 accumulates in the trailing edge of B16-F1 cells (Fig. 9a). Our hypothesis is further supported by the ability of IQGAP1 to crosslink actin filaments, as well as the presence of a $\mathrm{PI}(4,5) \mathrm{P}_{2}$-binding site as found in $\mathrm{CtxI}^{43,44,38}$. It is quite possible, however, that in vertebrates crosslinking and membrane attachment of cortical actin filament is additionally supported by other crosslinkers, for instance the $\mathrm{PI}(4,5) \mathrm{P}_{2}$-activated ERM 
proteins ${ }^{59}$. Importantly, for the first time, we show localization of active mDial in the rear cortex of migrating cells. mDial colocalized together with cortical F-actin and myosin IIA, thus suggesting a critical role in rear cortex function (Fig. 8b). This is consistent with the blebbing phenotype of mDial-depleted cells and enrichment of mDial at the cell rear of invading cells during entosis ${ }^{23,60}$. Moreover, mDial-depleted cells were previously shown to be impaired in polarization, migration and chemotaxis $^{61}$. mDial shares several main features with ForA, suggesting that this DRF and ForA represent functional homologues: (i) it is localized to the rear of migrating cells; (ii) the interaction of $\mathrm{N}$-terminal residues with $\mathrm{PI}(4,5) \mathrm{P}_{2}$ is required but not sufficient for membrane targeting and it additionally requires RhoA signalling; (iii) it dynamically altered its position in turning B16-F1 cells (Fig. 9c). Despite the fact that mDial is one of the best characterized formins in the field, it is rather surprising why its prominent rear localization in migrating cells has not been discovered previously. We assume that this may be due to the use of different cell types, overexpression or transfections with $\mathrm{N}$-terminally truncated constructs lacking $\mathrm{PI}(4,5) \mathrm{P}_{2}$ or the GTPase-interaction surfaces.

\section{Methods}

Plasmids. For expression of ForA GFP fusion proteins, first ForA-N (aa 1-647) was amplified by PCR as a BamHI/SalI fragment using complementary DNA from AX2 wild-type cells as template (European Nucleotide Archive accession number LN870236), and cloned into the BamHI/SalI sites of pDGFP-MCS-Neo ${ }^{27}$. FL ForA (aa 1-1,218) was generated by insertion of the remaining coding sequence of the forA gene into the SpeI/SalI sites of GFP-ForA-N. GFP-ForA $\triangle D A D$ (aa 1-1,138) and GFP-ForA $\triangle \mathrm{C} 2 \triangle \mathrm{DAD}$ (aa 91-1,138) were obtained by PCR using ForA-FL as a template, the fragments were digested with BamHI/SalI and inserted into the corresponding sites of pDGFP-MCS-Neo. For expression of GFP-tagged C2, a fragment corresponding to residues 1-99 was amplified by PCR and inserted into pDGFP-MCS-Neo. For expression of ForA constructs in Escherichia coli ForA-FH3 (aa 139-539), ForA-FH3L (aa 91-647), ForA-C2 (aa 1-99) and ForA-C (aa 635-1,218) were amplified by PCR and inserted into the BamHI and SalI sites of pGEX-6P-1 (GE Healthcare). For expression of MBP-GBD-PAK1, the coding sequences corresponding to residues 57-200 of rat PAK1 complementary DNA were amplified by PCR and inserted into the BamHI/SalI sites of pMAL-c2X (NEB). The plasmids for expression of GST- and GFP-tagged RaclA have been reported ${ }^{42}$. The for $A$ knockout vector was constructed by amplification of a 750-base pair $5^{\prime}$ PstI/BamHI fragment and a 900-base pair $3^{\prime}$ SalI/HindIII fragment of the for A gene from genomic DNA, respectively, and insertion of the fragments into the corresponding sites of pLPBLP ${ }^{62}$. Primer sequences of all constructs are provided in Supplementary Table 1 . The sequences of all constructs were verified by sequencing. The plasmid for expression of GFP-tagged myosin-II heavy chain was pBigGFPmyo $^{63}$. For transfection of B16-F1 mouse melanoma cells, mDia1 $\Delta \mathrm{DAD}^{56}$ was used. EGFP-tagged IQGAP1 was provided by Addgene (Plasmid \#30112).

Cell culture and transfections. D. discoideum cells were grown in HL5-C medium including glucose (Formedium) and were transformed by electroporation with an Xcell gene pulser (Bio-Rad) using pre-set protocol 4-6 for Dictyostelium. The for $A^{-}$and $m h c A^{-} /$for $A^{-}$cells were obtained by disruption of the for $A$ gene in AX2 wild-type cells and in the $m h c A^{-}$mutant $^{24}$, respectively, by homologous recombination using the pLPBLP-based targeting vector system ${ }^{62}$ after digestion of the targeting vector with BamHI and SalI. Stably transfected cells were selected with $10 \mu \mathrm{g} \mathrm{ml}^{-1}$ blasticidin S (Invivogen). Gene disruption was initially screened by PCR and eventually confirmed by the absence of the protein in immunoblots. Cell lines expressing GFP fusion proteins were obtained by transfecting the cells with the appropriate plasmids and selecting the transformants with $10 \mu \mathrm{g} \mathrm{ml}^{-1}$ of geneticin (Sigma). B16-F1 mouse melanoma cells (ATTC CRL-6323) were grown in high-glucose DMEM (Biowest) supplemented with 10\% FCS (Biowest) and $2 \mathrm{mM}$ glutamine (Sigma). Transfections were performed overnight using JetPrime (Polyplus) according to manufacturers' instructions.

Protein purification. Expression of GST- or MBP-tagged proteins was induced in E. coli strain Rossetta 2 (Novagen) with $1 \mathrm{mM}$ isopropyl-b-D-thiogalactoside at $21^{\circ} \mathrm{C}$ for $12 \mathrm{~h}$. The proteins were subsequently purified from bacterial extracts by affinity chromatography using either glutathione-conjugated agarose (Sigma-

Aldrich) or amylose high-flow resin (NEB) followed by size-exclusion chromatography on an Äkta Purifier System equipped with a HiLoad 26/600 Superdex 200 column (GE Healthcare). In case of GST-ForA-C, before size-exclusion chromatography, the GST-tag was cleaved by PreScission protease (GE Healthcare), and uncleaved GST-ForA-C and GST were removed by a second affinity chromatography using glutathione-conjugated agarose. The purification of heterodimeric capping protein $(\mathrm{CP})^{56}, \mathrm{mDial}^{-\mathrm{C}^{56}}$ and Dictyostelium PFN I was performed accordingly. The purified proteins were dialysed against storage buffer $(100 \mathrm{mM} \mathrm{KCl}, 1 \mathrm{mM}$ dithiothreitol (DTT), 60\% glycerol and $20 \mathrm{mM}$ HEPES pH 7.4) and stored at $-20^{\circ} \mathrm{C}$ for later use. Actin was purified from acetone powder of rabbit skeletal muscle according to standard procedures and labelled on Cys374 with ATTO 488 maleimide (ATTO-TEC) or with $N$-(1-Pyrenyl)maleimide (Invitrogen), respectively.

Actin biochemistry. The polymerization of $2 \mu \mathrm{M}$ rabbit skeletal muscle G-actin (5\% pyrene labelled) was initiated with $1 \times \mathrm{KMEI}(50 \mathrm{mM} \mathrm{KCl}, 1 \mathrm{mM} \mathrm{MgCl}$, $1 \mathrm{mM}$ EGTA and $10 \mathrm{mM}$ imidazol, $\mathrm{pH}$ 7.0) and was monitored in 96-well plates either alone or in the presence of various concentrations of ForA-C using a Synergy 4 fluorescence microplate reader (Biotek). To quantify formin autoinhibition, $50 \mathrm{nM}$ of ForA-C were incubated with various concentrations of GST-tagged ForA$\mathrm{N}$ in $1 \times \mathrm{KMEI}$ for $2 \mathrm{~min}$. Subsequently, $2 \mu \mathrm{M}$ G-actin were added and the polymerization rates were recorded as above. The maximum slopes of the polymerization curves were normalized, averaged for each data set and plotted against the molar ratio of ForA-N/ForA-C. As $50 \mathrm{nM}$ ForA-C was within the range during which the polymerization rate increased linearly to the concentration of ForA-C and the slopes indicated high-affinity binding of ForA-N to ForA-C, the data were fitted by linear regression. The intersection of the lines marks the molar ratio required for complete inhibition.

For assaying dilution-induced depolymerization experiments, G-actin (50\% pyrene labelled) was first polymerized in $1 \times \mathrm{KMEI}$ for $2 \mathrm{~h}$ at room temperature. Subsequently, the F-actin was preincubated with various concentrations of ForA-C or CP. The depolymerization reaction was initiated by diluting F-actin to $0.1 \mu \mathrm{M}$ by addition $1 \times$ KMEI buffer into the wells using the automated dispenser of the Synergy 4 plate reader and analysed as above. For in vitro TIRF microscopy, ForA$\mathrm{C}(10$ or $100 \mathrm{nM}$ final concentration) and Dictyostelium PFN I $(5 \mu \mathrm{M}$ final concentration) were pre-diluted in $1 \times$ TIRF buffer $(20 \mathrm{mM}$ imidazole $\mathrm{pH} 7.4$, $50 \mathrm{mM} \mathrm{KCl}, 1 \mathrm{mM} \mathrm{MgCl}$, $1 \mathrm{mM}$ EGTA, $20 \mathrm{mM} \beta$-mercaptoethanol, $0.5 \mathrm{mM}$ ATP, $15 \mathrm{mM}$ glucose, $2.5 \mathrm{mg} \mathrm{ml}^{-1}$ methylcellulose $(4,000 \mathrm{cP}), 20 \mu \mathrm{g} \mathrm{ml}^{-1}$ catalase and $100 \mu \mathrm{g} \mathrm{ml}^{-1}$ glucose oxidase). The assays were initiated by adding G-actin ( $1 \mu \mathrm{M}$ final concentration, $10 \%$ Atto488 labelled) and flushing the mixtures into mPEGsilane MW 2000 (Laysan Bio) pre-coated flow chambers. Images were captured with a Nikon Eclipse TI-E inverted microscope equipped with a TIRF Apo $\times 60$ or $\times 100$ objective at 1 -s intervals with exposure times of $40 \mathrm{~ms}$ by a Ixon 3897 EMCCD camera (Andor) for at least $10 \mathrm{~min}$. The pixel size corresponded to $0.159 \mu \mathrm{m}$ for the $\times 100$ objective and $0.27 \mu \mathrm{m}$ for the $\times 60$ objective. The nucleation efficacies were obtained by counting and averaging the number of actin filaments in an area of $80 \times 80 \mu \mathrm{m}$ after $180 \mathrm{~s}$ in three independent experiments $(n=3)$. The elongation rates of filaments were determined by manual tracking of growing barbed ends using ImageJ software. At least 20 filaments from three movies per condition were measured for the experiments in presence of PFN I.

Antibodies. Polyclonal antibodies against ForA and Rac1A were raised by immunizing a female New Zealand white rabbit with either recombinant GSTtagged ForA-FH3L or GST-tagged RaclA following standard protocols. Subsequently, the polyclonal antibodies were affinity purified after coupling of the same antigens to $\mathrm{CNBr}$-activated Sepharose (GE Healthcare). Immunoblotting was performed according to standard protocols using undiluted hybridoma supernatants of IQGAP1-specific mAb 216-394-1 (ref. 30, GFP-specific mAb 264-449-2 (ref. 30), myosin-II-specific mAb 56-396-5 (ref. 64), Porin-specific mAb 70-100-1 (ref. 65) or polyclonal anti-Rac1A and anti-ForA antibodies (1:250 dilution). Alexa488-conjugated nanobodies were from Chromotek (1:200 dilution; \#gba488) and polyclonal anti-myosin IIA antibodies were from Thermo Scientific (1:100 dilution; \#PA5-17025). Primary antibodies in immunoblots were visualized with phosphatase-coupled anti-mouse (1:1,000, \#115-055-62; Dianova) or anti-rabbit IgG (1:1,000; \#111-055-046; Dianova). Uncropped scans of the most important immunoblots are shown in Supplementary Fig. 6. For immunohistochemistry, secondary Alexa-555-conjugated goat-anti-rabbit polyclonal antibodies (1:1,000 dilution; \#A21429; Invitrogen) were used.

Fluorescence microscopy and imaging. For immunofluorescence labelling of Dictyostelium cells, growth-phase cells expressing GFP-tagged proteins were washed twice with PB, and allowed to adhere on glass coverslips for $20 \mathrm{~min}$. The cells were then fixed with picric acid/paraformaldehyde, permebealized with $70 \%$ ethanol, washed extensively with PBS containing $2.7 \mathrm{mM} \mathrm{KCl}, 1.8 \mathrm{mM} \mathrm{KH}_{2} \mathrm{PO}_{4}$, $10 \mathrm{mM} \mathrm{Na}_{2} \mathrm{HPO}_{4}, 140 \mathrm{mM} \mathrm{NaCl}, \mathrm{pH} 7.3$ and labelled for F-actin with TRITCconjugated phalloidin (1:200 dilution, \#P1951; Sigma). The GFP signal was enhanced by Alexa488-conjugated nanobodies. Fixation of cells compressed under agar was performed accordingly. For live cell imaging, growth-phase cells expressing GFP-tagged proteins were seeded onto 3-cm-diameter glass-bottom dishes (Matek) and allowed to adhere on the glass surface for $20 \mathrm{~min}$. The cells were then washed four times with PB and imaged using an LSM510Meta confocal microscope (Zeiss) equipped with a $\times 63 / 1.3$ Plan-Neofluar objective using the $488 \mathrm{~nm}$ and $543 \mathrm{~nm}$ laser lines. 
For indirect immunofluorescence microscopy, B16-F1 cells were plated on acidwashed coverslips coated with $\mu \mathrm{g} \mathrm{ml}^{-1}$ laminin (Sigma). After fixation with $4 \%$ paraformaldehyde in PBS for $15 \mathrm{~min}$, the specimens were permebealized with PBS containing $100 \mathrm{mM}$ glycine and $0.1 \%$ Triton X-100 for $5 \mathrm{~min}$. After extensive washing with PBS containing $0.05 \%(\mathrm{v} / \mathrm{v})$ cold fish gelatine (Sigma) and $0.5 \%(\mathrm{w} / \mathrm{v})$ bovine serum albumin (Sigma), the cells were stained with the probes indicated. For live cell imaging, the transfected B16-F1 cells were plated onto 3-cm-diameter glass-bottom dishes (Matek) coated with $25 \mu \mathrm{g} \mathrm{ml}^{-1}$ laminin (Sigma), mounted in a heated miniature incubator (Bioscience Tools) and observed at $37^{\circ} \mathrm{C}$ with an Olympus IX-81 inverted microscope equipped with a $\times 40 / 0.75$ numerical aperture UplanFL objective and a cooled CCD camera (CoolSNAP EZ, Photometrics) with a filter wheel and shutters controlled by Metamorph software (Molecular Devices). Data were processed using ImageJ, Adobe Photoshop CS (Adobe Systems) and CorelDraw software (Corel Corporation).

Analyses of cell migration. Quantitative analysis of random cell motility was performed as follows: growth-phase cells in PB buffer were monitored every $10 \mathrm{~s}$ for $15 \mathrm{~min}$ by time-lapse imaging at an inverted Olympus IX-81 equipped with $\times 10$ phase-contrast optics (Olympus) and a CoolSnap EZ camera (Photometrics). Migration assays in 2D-confined environments were performed with growth-phase cells that were overlaid with a $0.17-\mathrm{mm}$-thin sheet of agar ( $2 \%$ in PB buffer). The cells were subsequently allowed to adapt for $90 \mathrm{~min}$ to the compression before imaging, which was expanded to $30 \mathrm{~min}$ in the motility assays. Single-cell tracks were obtained from recordings with the Track Objects plugin of Metamorph 7 software (Molecular Devices). Data samples of each cell line contain at least 60 individual cells from at least three different experiments. These data were further processed to obtain average speed of single cells in a custom-built protocol operated in Excel (Microsoft). Average speed distributions of analysed samples are presented in box plot representations using SigmaPlot 11.2 (Systat Software Inc). Boxes indicate $25-75$ percentiles, the whiskers mark the 10th and 90th, and the outliers the 5th and 95th percentile. Red dashed lines indicate mean values. Centroid velocities were determined from pseudo-phase-contrast time-lapse recordings using the ImageJ plugin BOA of the QuimP11b plugin suite ${ }^{66}$. The instantaneous centroid velocity to a given time point $t$ was approximated by calculation of simple moving averages (SMAs) comprising velocities of the previous and following time point amounting to velocity $\mathrm{t}_{\mathrm{SMA}}=\left(\right.$ velocity $_{\mathrm{t}-1}+$ velocity $_{\mathrm{t}}+$ velocity $\left._{\mathrm{t}+1}\right) / 3$. The size of the posterior blebs was determined using the selection and measurement tools in Image software.

Analyses of cortical ForA distribution in migrating and repolarizing cells. Protrusion and retraction velocities as well as cortical ForA fluorescence intensities were determined by two custom-built macros for ImageJ software and protocols operated in Excel. The radial displacement of the cell edge between the time point $t$ and a former time point $t-1$ was measured as follows: the cell boundaries of both time points were manually outlined from confocal fluorescence images as two pixel-wide lines onto blank images of the same size. Using the first custom-built macro, the radial distance between the cell centroid at $t$ and the outlined cell edge was automatically determined by line scans at each angle relative to the direction of movement (defined by the cell centroids at $t$ and $t-1$ and set as $0^{\circ}$, Supplementary Fig. 2a). For this purpose, the macro employs the commands 'plot', 'profile' and 'rotate'. The net displacement of the cell boundary at each angle corresponds to the difference of the boundary distances at $t-1$ and $t$ and was calculated in Excel. ForA fluorescence intensity profiles at each angle were generated by the second macro (Supplementary Fig. 2b). The average of the three brightest pixels at the determined cortical position was extracted from the profile data by a custom-built Excel protocol. The resulting angular profiles correlating protrusion and retraction rates with ForA fluorescence intensity before, during and after repolarization are exemplarily shown in Supplementary Fig. 2c.

Micropipette aspiration. For the micropipette aspiration, a chamber having one open side was filled with PB buffer and mounted on a stage of an inverted Axiovert 200 microscope (Zeiss) equipped with a LD Achroplan $\times 40 / 0.6$ objective (Zeiss). The position of the micropipettes was controlled by a SM 3.25 micromanipulator (Märzhäuser Wetzlar). Aspiration pressure was applied by a height-adjustable water reservoir driven by a piezoelectric magnet slider and was calibrated with $2-\mu \mathrm{m}$ polystyrene beads (Polysciences) to obtain $0 \mathrm{~Pa}$. For the experiments, glass capillaries with an inner diameter of $3.4 \pm 0.8 \mu \mathrm{m}$ were used. After setting the pressure difference to 500 or $1,250 \mathrm{~Pa}$, PB buffer washed growth-phase cells were carefully injected into the chamber, and only non-adhering cells aspirated. The aspirations were recorded by differential interference contrast (DIC) imaging using a Sensicam CCD camera (PCO) with a frame rate of at least $2 \mathrm{~Hz}$. Data were analysed by Fiji software.

Lipid co-floatation assays. 1-Palmitoyl-2-oleoyl-sn-glycero-3-phosphatidylcholine (POPC), 1-palmitoyl-2-oleoyl-sn-glycero-3-phosphatidylethanolamine (POPE), 1-palmitoyl-2-oleoyl-sn-glycero-3-phosphatidylserine (POPS), phosphatidyl-inositol-(3)-phosphate, phosphatidyl-inositol-(4)-phosphate, phosphatidylinositol-(3,5)-bisphosphate, $\mathrm{PI}(4,5) \mathrm{P}_{2}$ and $\mathrm{PI}(3,4,5) \mathrm{P}_{3}$ were purchased from Avanti Polar Lipids. Lipid compositions were POPC:POPE:POPS at a ratio of 6:2:2 for the carrier lipids and POPC:POPE:POPS:PIPx at a ratio of 5:2:2:1. Lipids in desired concentrations were mixed and then dried under a stream of nitrogen. The lipids were subsequently maintained under reduced pressure for at least $3 \mathrm{~h}$, and subsequently dissolved in $20 \mathrm{mM}$ HEPES buffer, $\mathrm{pH} 7.4,100 \mathrm{mM} \mathrm{NaCl}$ and $0.3 \mathrm{M}$ sucrose with occasional gentle vortexing for $1 \mathrm{~h}$ at room temperature to form liposomes. For visualization, liposomes were labelled with 5\% rhodamine B-conjugated PE (Avanti polar lipids). A mixture of GST-ForA-C2 $(1.72 \mu \mathrm{M})$ and liposomes (total lipid $166.67 \mu \mathrm{M}$ ) was incubated at room temperature for $15 \mathrm{~min}$ Samples were then brought to $30 \%$ sucrose in HEPES buffer by gentle mixing (final volume $250 \mu \mathrm{l}$ ) and overlaid with $200 \mu \mathrm{l}$ each containing either $25 \%$ sucrose or $0 \%$ sucrose solution in the above described HEPES buffer. The samples were centrifuged at 50,000 r.p.m. for $30 \mathrm{~min}$ at $4{ }^{\circ} \mathrm{C}$ with a Beckman Optima Max ultracentrifuge using a TLS55 rotor. Fractions of $100 \mu \mathrm{l}$ were collected from top (fraction 1 ) to bottom (fraction 4 ), and $25-\mu 1$ samples analysed by SDSpolyacrylamide gel electrophoresis and Coomassie blue staining. Intensities of protein bands in fraction 2 were quantified by densitometry using the Quantity One software (Bio-Rad), and are expressed as percentage of the total protein.

GTPase activation assays. For Rac1-GTP-binding assays, $200 \mu \mathrm{g}$ of MBP fused to PAK-CRIB was immobilized on amylose high-flow resin (NEB). Cells grown in 10-cm-diameter dishes were harvested by centrifugation, washed twice with cold PB buffer (17 mM Na-K-phosphate, pH 6.0) and lysed with $1 \mathrm{ml}$ of ice-cold lysis buffer ( $25 \mathrm{mM}$ Tris, pH 7.5, $10 \mathrm{mM} \mathrm{MgCl}, 50 \mathrm{mM} \mathrm{NaCl}, 2 \mathrm{mM}$ EGTA, $2 \mathrm{mM}$ DTT, $1 \% n$-octylpolyoxyethylene (Bachem), $2 \mathrm{mM}$ benzamidine, bestatin $\left(0.5 \mathrm{mg} \mathrm{ml}^{-1}\right)$, and pepstatin, antipain and leupeptin (each $1 \mathrm{mg} \mathrm{ml}^{-1}$ ). After centrifugation at $15,000 \mathrm{~g}$ for $10 \mathrm{~min}, 900 \mu \mathrm{l}$ of the lysates each were added to the beads and incubated for $60 \mathrm{~min}$ at $4^{\circ} \mathrm{C}$ on a rotary wheel. After the beads were washed three times with wash buffer $(25 \mathrm{mM}$ Tris, $\mathrm{pH} 7.5,10 \mathrm{mM} \mathrm{MgCl} 2,50 \mathrm{mM}$ $\mathrm{NaCl}, 2 \mathrm{mM}$ DTT and $0.1 \% n$-octylpolyoxyethylene), the precipitates were analysed by immunoblotting with polyclonal anti-Racl antibodies. For quantification of relative Rac1-GTP levels, band intensities were measured by densitometry and analysed using ImageJ software. Band intensities of active Rac1 from four independent experiments were averaged and normalized to wild-type levels.

Statistical analysis. Statistical analysis was performed using SigmaPlot 11.2 software (Systat Software Inc.). Statistical significance of differences between nonnormally distributed populations was determined by the Mann-Whitney $U$-test. When data fulfilled the criteria of normality (Shapiro-Wilk test) and equal variance (Levene's test), statistical differences were analysed with a two-tailed, unpaired Student's $t$-test. Statistical differences are reported as ${ }^{\star} P \leq 0.05,{ }^{*} P \leq 0.01$, ${ }^{* * *} P \leq 0.001$ and NS as not significant.

\section{References}

1. Friedl, P. \& Wolf, K. Plasticity of cell migration: a multiscale tuning model. J. Cell Biol. 188, 11-19 (2010).

2. Trepat, X., Chen, Z. \& Jacobson, K. Cell migration. Compr. Physiol. 2, 2369-2392 (2012).

3. Roubinet, C., Tran, P. T. \& Piel, M. Common mechanisms regulating cell cortex properties during cell division and cell migration. Cytoskeleton 69, 957-972 (2012).

4. Bear, J. E. \& Haugh, J. M. Directed migration of mesenchymal cells: where signaling and the cytoskeleton meet. Curr. Opin. Cell Biol. 30, 74-82 (2014).

5. Lämmermann, T. \& Sixt, M. Mechanical modes of 'amoeboid' cell migration. Curr. Opin. Cell Biol. 21, 636-644 (2009).

6. Tyson, R. A., Zatulovskiy, E., Kay, R. R. \& Bretschneider, T. How blebs and pseudopods cooperate during chemotaxis. Proc. Natl Acad. Sci. USA 111, 11703-11708 (2014).

7. Cramer, L. P. Forming the cell rear first: breaking cell symmetry to trigger directed cell migration. Nat. Cell Biol. 12, 628-632 (2010).

8. Yam, P. T. et al. Actin-myosin network reorganization breaks symmetry at the cell rear to spontaneously initiate polarized cell motility. J. Cell Biol. 178, 1207-1221 (2007).

9. Verkhovsky, A. B., Svitkina, T. M. \& Borisy, G. G. Self-polarization and directional motility of cytoplasm. Curr. Biol. 9, 11-20 (1999).

10. Swaney, K. F., Huang, C. H. \& Devreotes, P. N. Eukaryotic chemotaxis: a network of signaling pathways controls motility, directional sensing, and polarity. Annu. Rev. Biophys. 39, 265-289 (2010).

11. Salbreux, G., Charras, G. \& Paluch, E. Actin cortex mechanics and cellular morphogenesis. Trends Cell Biol. 22, 536-545 (2012).

12. Merkel, R. et al. A micromechanic study of cell polarity and plasma membrane cell body coupling in Dictyostelium. Biophys. J. 79, 707-719 (2000).

13. Vicente-Manzanares, M. et al. Myosin IIA/IIB restrict adhesive and protrusive signaling to generate front-back polarity in migrating cells. J. Cell Biol. 193, 381-396 (2011).

14. Bretscher, A. Microfilament structure and function in the cortical cytoskeleton. Ann. Rev. Cell Biol 7, 337-374 (1991).

15. Stewart, M. P. et al. Hydrostatic pressure and the actomyosin cortex drive mitotic cell rounding. Nature 469, 226-230 (2011).

16. Munjal, A. \& Lecuit, T. Actomyosin networks and tissue morphogenesis. Development 141, 1789-1793 (2014). 
17. Pollard, T. D. Regulation of actin filament assembly by Arp $2 / 3$ complex and formins. Annu. Rev. Biophys. Biomol. Struct. 36, 451-477 (2007).

18. Vinzenz, M. et al. Actin branching in the initiation and maintenance of lamellipodia. J Cell Sci. 125, 2775-2785 (2012).

19. Eisenmann, K. M. et al. Dia-interacting protein modulates formin-mediated actin assembly at the cell cortex. Curr. Biol. 17, 579-591 (2007).

20. Hannemann, S. et al. The Diaphanous-related Formin FHOD1 associates with ROCK1 and promotes Src-dependent plasma membrane blebbing. J. Biol. Chem. 283, 27891-27903 (2008).

21. Kitzing, T. M., Wang, Y., Pertz, O., Copeland, J. W. \& Grosse, R. Formin-like 2 drives amoeboid invasive cell motility downstream of RhoC. Oncogene 29, 2441-2448 (2010).

22. Stastna, J. et al. Differing and isoform-specific roles for the formin DIAPH3 in plasma membrane blebbing and filopodia formation. Cell Res. 22, 728-745 (2012).

23. Bovellan, M. et al. Cellular control of cortical actin nucleation. Curr. Biol. 24, 1628-1635 (2014).

24. Wessels, D. et al. Cell motility and chemotaxis in Dictyostelium amoebae lacking myosin heavy chain. Dev. Biol. 128, 164-177 (1988).

25. Kay, R. R., Langridge, P., Traynor, D. \& Hoeller, O. Changing directions in the study of chemotaxis. Nat. Rev. Mol. Cell Biol. 9, 455-463 (2008).

26. Kovar, D. R., Harris, E. S., Mahaffy, R., Higgs, H. N. \& Pollard, T. D. Control of the assembly of ATP- and ADP-actin by formins and profilin. Cell 124, 423-435 (2006).

27. Dumontier, M., Höcht, P., Mintert, U. \& Faix, J. Racl GTPases control filopodia formation, cell motility, endocytosis, cytokinesis and development in Dictyostelium. J. Cell Sci. 113, 2253-2265 (2000).

28. Faix, J. et al. Cortexillins, major determinants of cell shape and size, are actinbundling proteins with a parallel coiled-coil tail. Cell 86, 631-642 (1996).

29. Weber, I. et al. Cytokinesis mediated through the recruitment of cortexillins into the cleavage furrow. EMBO J. 18, 586-594 (1999).

30. Faix, J. et al. Recruitment of cortexillin into the cleavage furrow is controlled by Rac1 and IQGAP-related proteins. EMBO J. 20, 3705-3715 (2001).

31. Mondal, S. et al. Regulation of the actin cytoskeleton by an interaction of IQGAP related protein GAPA with filamin and cortexillin I. PLoS ONE 5, e15440 (2010).

32. Simson, R. et al. Membrane bending modulus and adhesion energy of wild-type and mutant cells of Dictyostelium lacking talin or cortexillins. Biophys. J. 74, 514-522 (1998).

33. Kee, Y. S. et al. A mechanosensory system governs myosin II accumulation in dividing cells. Mol. Biol. Cell 23, 1510-1523 (2012).

34. Burke, T. A. et al. Homeostatic actin cytoskeleton networks are regulated by assembly factor competition for monomers. Curr. Biol. 24, 579-585 (2014).

35. Neujahr, R. et al. Three-dimensional patterns and redistribution of myosin II and actin in mitotic Dictyostelium cells. J. Cell Biol. 139, 1793-1804 (1997).

36. Charras, G. \& Paluch, E. Blebs lead the way: how to migrate without lamellipodia. Nat. Rev. Mol. Cell Biol. 9, 730-736 (2008).

37. Rizo, J. \& Südhof, T. C. C2-domains, structure and function of a universal Ca2 + -binding domain. J. Biol. Chem. 273, 15879-15882 (1998).

38. Stock, A. et al. Domain analysis of cortexillin I: actin-bundling, $\mathrm{PIP}_{2}$-binding and the rescue of cytokinesis. EMBO J. 18, 5274-5384 (1999).

39. Filić, V., Marinović, M., Faix, J. \& Weber, I. A dual role for Rac1 GTPases in the regulation of cell motility. J. Cell Sci. 125, 387-398 (2012).

40. Steffen, A. et al. Rac function is crucial for cell migration but is not required for spreading and focal adhesion formation. J. Cell Sci. 126, 4572-4588 (2013).

41. Lee, S., Shen, Z., Robinson, D. N., Briggs, S. \& Firtel, R. A. Involvement of the cytoskeleton in controlling leading-edge function during chemotaxis. Mol. Biol. Cell 21, 1810-1824 (2010).

42. Faix, J. et al. The IQGAP-related protein DGAP1 interacts with Rac and is involved in the modulation of the F-actin cytoskeleton and control of cell motility. J. Cell Sci. 111, 3059-3071 (1998).

43. Bashour, A. M., Fullerton, A. T., Hart, M. J. \& Bloom, G. S. IQGAP1, a Rac- and Cdc42-binding protein, directly binds and cross-links microfilaments. J. Cell Biol. 137, 1555-1566 (1997).

44. Choi, S. et al. IQGAP1 is a novel phosphatidylinositol 4,5 bisphosphate effector in regulation of directional cell migration. EMBO J. 32, 2617-2630 (2013).

45. Foroutannejad, S., Rohner, N., Reimer, M., Kwon, G. \& Schober, J. M. A novel role for IQGAP1 protein in cell motility through cell retraction. Biochem. Biophys. Res. Commun. 448, 39-44 (2014).

46. Ren, J. G., Li, Z., Crimmins, D. L. \& Sacks, D. B. Self-association of IQGAP1: characterization and functional sequelae. J. Biol. Chem. 280, 34548-34557 (2005).

47. Watanabe, N., Kato, T., Fujita, A., Ishizaki, T. \& Narumiya, S. Cooperation between $\mathrm{mDia} 1$ and ROCK in Rho-induced actin reorganization. Nat. Cell Biol. 1, 136-143 (1999)
48. Charras, G. T., Yarrow, J. C., Horton, M. A., Mahadevan, L. \& Mitchison, T. J. Non-equilibration of hydrostatic pressure in blebbing cells. Nature 435, 365-369 (2005).

49. Poincloux, R. et al. Contractility of the cell rear drives invasion of breast tumor cells in 3D Matrigel. Proc. Natl Acad. Sci. USA 108, 1943-1948 (2011).

50. Yoshida, K. \& Soldati, T. Dissection of amoeboid movement into two mechanically distinct modes. J Cell Sci. 119, 3833-3844 (2006).

51. Filić, V., Marinović, M., Faix, J. \& Weber, I. The IQGAP-related protein DGAP1 mediates signaling to the actin cytoskeleton as an effector and a sequestrator of Rac1 GTPases. Cell. Mol. Life Sci. 71, 2775-2785 (2014).

52. Maiuri, P. et al. Actin flows mediate a universal coupling between cell speed and cell persistence. Cell 161, 374-386 (2015).

53. Martinelli, S. et al. Ezrin/radixin/moesin proteins and flotillins cooperate to promote uropod formation in T cells. Front. Immunol. 8, 84 (2013).

54. Fukui, Y., Kitanishi-Yumura, T. \& Yumura, S. Spatiotemporal dynamics of actin concentration during cytokinesis and locomotion in Dictyostelium. J. Cell Sci. 111, 2097-2108 (1998).

55. Wessels, D., Lusche, D. F., Kuhl, S., Heid, P. \& Soll, D. R. PTEN plays a role in the suppression of lateral pseudopod formation during Dictyostelium motility and chemotaxis. J. Cell Sci. 120, 2517-2531 (2007).

56. Ramalingam, N. et al. Phospholipids regulate localization and activity of mDial formin. Eur. J. Cell Biol. 89, 723-732 (2010).

57. Seth, A., Otomo, C. \& Rosen, M. K. Autoinhibition regulates cellular localization and actin assembly activity of the diaphanous-related formins FRLalpha and mDia1. J. Cell Biol. 174, 701-713 (2006).

58. Witze, E. S. et al. Wnt5a directs polarized calcium gradients by recruiting cortical endoplasmic reticulum to the cell trailing edge. Dev. Cell 26, 645-657 (2013).

59. Clucas, J. \& Valderrama, F. ERM proteins in cancer progression. J. Cell Sci. 127, 267-275 (2014).

60. Purvanov, V., Holst, M., Khan, J., Baarlink, C. \& Grosse, R. G-protein-coupled receptor signaling and polarized actin dynamics drive cell-in-cell invasion. Elife 20, 3 (2014).

61. Shi, Y. et al. The mDial formin is required for neutrophil polarization, migration, and activation of the LARG/RhoA/ROCK signaling axis during chemotaxis. J. Immunol. 182, 3837-3845 (2009).

62. Faix, J., Kreppel, L., Shaulsky, G., Schleicher, M. \& Kimmel, A. R. A rapid and efficient method to generate multiple gene disruptions in Dictyostelium discoideum using a single selectable marker and the Cre-loxP system. Nucleic Acids Res. 32, e143 (2004).

63. Moores, S. L., Sabry, J. H. \& Spudich, J. A. Myosin dynamics in live Dictyostelium cells. Proc. Natl Acad. Sci. USA 93, 443-446 (1996).

64. Pagh, K. \& Gerisch, G. Monoclonal antibodies binding to the tail of Dictyostelium discoideum myosin: their effects on antiparallel and parallel assembly and actin-activated ATPase activity. J. Cell Biol. 103, 1527-1538 (1986).

65. Troll, H. et al. Purification, functional characterization, and cDNA sequencing of mitochondrial porin from Dictyostelium discoideum. J. Biol. Chem. 267, 21072-21079 (1992).

66. Bosgraaf, L., van Haastert, P. J. \& Bretschneider, T. Analysis of cell movement by simultaneous quantification of local membrane displacement and fluorescent intensities using Quimp2. Cell Motil. Cytoskeleton. 66, 156-165 (2009).

\section{Acknowledgements}

We thank Daniela Rieger and Annette Breskott for technical assistance and Klemens Rottner for helpful comments on the manuscript. We further thank Annette MüllerTaubenberger and Günther Gerisch for porin antibodies. This work was supported by the Deutsche Forschungsgemeinschaft (FA 330/6-2, FA 330/9-1 and FA 330/10-1 to J.F., and SCHL 204/11-1 to M.S.) and the Elite Network Bavaria to N.R. H.Z. was supported by grants from the Academy of Finland (funding decision no. 266846 and 273650).

\section{Author contributions}

J.F. designed the experiments, interpreted the data and wrote the paper. N.R. and C.F. generated DNA constructs, mutants, ForA antibodies and performed confocal imaging and analyses of cell migration. E.J. performed aspiration assays. M.W. and S.B. purified proteins, performed pyrene assays and TIRF experiments. Y.L. and H.Z. performed and analysed lipid-binding assays. A.J. developed Racl antibodies and performed GTPase activation assays. H.M. expressed and purified N-terminal ForA constructs. A.A.N., I.W., M.S. and R.M. contributed to data interpretation and experimental design, as well as the development of the final version of the paper. All authors provided intellectual input to this work.

\section{Additional information}

Supplementary Information accompanies this paper at http://www.nature.com/ naturecommunications 
Competing financial interests: The authors declare no competing financial interests.

Reprints and permission information is available online at http://www.nature.com/ naturecommunications/

How to cite this article: Ramalingam, N. et al. A resilient formin-derived cortical actin meshwork in the rear drives actomyosin-based motility in $2 \mathrm{D}$ confinement. Nat. Commun. 6:8496 doi: 10.1038/ncomms9496 (2015). (c) (i) This work is licensed under a Creative Commons Attribution 4.0 International License. The images or other third party material in this article are included in the article's Creative Commons license, unless indicated otherwise in the credit line; if the material is not included under the Creative Commons license, users will need to obtain permission from the license holder to reproduce the material. To view a copy of this license, visit http://creativecommons.org/ licenses/by/4.0/ 